\title{
Boltzmann and Fokker-Planck Equations Modelling the Elo Rating System with Learning Effects
}

\author{
Bertram Düring $^{1}$ (D) Marco Torregrossa ${ }^{2} \cdot$ Marie-Therese Wolfram $^{3,4}$ \\ Received: 18 June 2018 / Accepted: 2 November 2018 / Published online: 22 November 2018 \\ (c) The Author(s) 2018
}

\begin{abstract}
In this paper, we propose and study a new kinetic rating model for a large number of players, which is motivated by the well-known Elo rating system. Each player is characterised by an intrinsic strength and a rating, which are both updated after each game. We state and analyse the respective Boltzmann-type equation and derive the corresponding nonlinear, non-local Fokker-Planck equation. We investigate the existence of solutions to the Fokker-Planck equation and discuss their behaviour in the long time limit. Furthermore, we illustrate the dynamics of the Boltzmann and Fokker-Planck equation with various numerical experiments.
\end{abstract}

Keywords Elo rating · Learning $\cdot$ Kinetic model $\cdot$ Fokker-Planck equation · Existence of weak solutions · Asymptotic behaviour

Mathematics Subject Classification 35Q91 · 35Q20 $\cdot 35 \mathrm{Q} 84 \cdot 35 \mathrm{~K} 65 \cdot 35 \mathrm{~B} 40 \cdot 91 \mathrm{G} 60$

Communicated by Paul Newton.

$凶$ Bertram Düring

bd80@sussex.ac.uk

Marco Torregrossa

marcotorr1986@gmail.com

Marie-Therese Wolfram

m.wolfram@warwick.ac.uk

1 Department of Mathematics, University of Sussex, Brighton BN1 9QH, UK

2 Department of Mathematics, University of Pavia, via Ferrata 1, 27100 Pavia, Italy

3 Mathematics Institute, University of Warwick, Coventry CV4 7AL, UK

4 Radon Institute of Computational and Applied Mathematics, Altenbergerstr. 69, 4040 Linz, Austria 


\section{Introduction}

In 1950, the Hungarian physicist Arpad Elo developed a rating system to calculate the relative skill level of players in competitor versus competitor games (see Elo (1978)). The Elo rating system was initially used in chess competitions, but was quickly adopted by the US Chess Federation as well as the World Chess Federation, and the National Football Foundation. In June 2018, FIFA announced switching their world football ranking to an Elo system, following two years of reviews and studies of different alternatives. The Elo rating system assigns each player a rating, which is updated according to the wins and losses as well as the difference of the ratings. It is hoped that the rating converges to the relative strength level and is a valid measure of the player's skills. However, assigning an initial rating to a new player is a delicate issue, since it is not clear how an inaccurate initial rating influences the latter performance. Elo himself tried to validate the model using computational experiments, while Glickman used statistical techniques to understand the dynamics (Glickman and Jones 1999). The first rigorous proof of convergence of the ratings to the individual strength was presented by in Jabin and Junca (2015), who introduced a continuous version of the Elo rating system. In this continuous model, every player is characterised by its intrinsic strength $\rho$ and rating $R$. The intrinsic strength is fixed in time. If two players with rating $R_{i}$ and $R_{j}$ meet in a game, their ratings after the game, $R_{i}^{*}$ and $R_{j}^{*}$ are given by

$$
\begin{aligned}
& R_{i}^{*}=R_{i}+K\left(S_{i j}-b\left(R_{i}-R_{j}\right)\right), \\
& R_{j}^{*}=R_{j}+K\left(-S_{i j}-b\left(R_{j}-R_{i}\right)\right) .
\end{aligned}
$$

In (1), the random variable $S_{i j}$ is the score result of the game; it takes the value 1 if player $i$ wins and the value -1 if player $j$ wins. The mean score (i.e. expected value of $\left.S_{i j}\right)$ is assumed to be equal to $b\left(\rho_{i}-\rho_{j}\right)$, and hence, the result of each game depends on the difference of the player's intrinsic strengths. The rating of each player increases or decreases proportionally with the outcome of the game, relative to the predicted mean score $b\left(R_{i}-R_{j}\right)$. The speed of the adjustment is controlled by the constant parameter $K$. The function $b$ is chosen in such a way that extreme differences are moderated; a typical choice is

$$
b(z)=\tanh (c z)
$$

where $c$ is a suitably chosen positive constant. This choice weighs the impact of the outcome with respect to the relative rating. If a player with a high rating wins a game against a player with a low rating, the players' ratings change little. However, if the player with the low rating wins against a highly rated player, the ratings are strongly adjusted.

Junca and Jabin proposed the following Boltzmann-type equation to describe the evolution of the distribution of players $f=f(r, t)$ with respect to their ratings

$$
\begin{array}{r}
\partial_{t} f(r, t)+\partial_{r}(a(f) f)=0 \text { with } a(f)=\int_{\mathbb{R}^{2}} w\left(r-r^{\prime}\right)\left(b\left(\rho-\rho^{\prime}\right)\right. \\
\left.-b\left(r-r^{\prime}\right)\right) f\left(t, r^{\prime}, \rho^{\prime}\right) \mathrm{d} \rho^{\prime} \mathrm{d} r^{\prime} .
\end{array}
$$


This equation describes a more general setup than in the microscopic equations. Here, two players only interact according to the interaction rate function $w$, which depends on the difference of their ratings. The function $w$ is assumed to be even and nonnegative. Junca and Jabin analysed the long-time behaviour of solutions to (3). They proved that in the case $w=1$, a so-called all-play-all tournament, the ratings converge exponentially fast to the intrinsic strength. In the case of local interactions, that is individuals only play if their ratings are close, the ratings may not converge to the intrinsic strength and the rating fails to give a fair representation of the player's strength distribution.

Rather recently Krupp (2016) proposed an extension of the model by Jabin and Junca (2015). In her model not only the rating, but also the intrinsic strength changes as players continuously compete in games. In particular, she assumes that the intrinsic strength $\rho$ changes in every game according to

$$
\begin{aligned}
& \rho_{i}^{*}=\rho_{i}+Z_{i j} \tilde{K}, \\
& \rho_{j}^{*}=\rho_{j}+Z_{i j} \tilde{K},
\end{aligned}
$$

where $\tilde{K}$ is a positive constant and $Z_{i j}$ takes the value $z_{1} \in \mathbf{N}$ or $z_{2} \in \mathbf{N}$. In case of a win, the inner strength $\rho_{i}$ increases by $z_{1} \tilde{K}$ and in case of a loss by $z_{2} \tilde{K}$. Hence, if $z_{1}<z_{2}$, the looser benefits more from the game, while if $z_{1}>z_{2}$, the winner learns more. If $z_{1}=z_{2}$, both learn the same. The corresponding Boltzmann-type equation for the distribution of the players $f=f(r, \rho, t)$ with respect to their strength and rating reads as

$$
\partial_{t} f(r, \rho, t)+\partial_{r}(a(f) f)+\partial_{\rho}(c(f) f)=0,
$$

where

$$
a(f)=\int_{\mathbb{R}^{2}} w\left(r-r^{\prime}\right)\left[b\left(\rho-\rho^{\prime}\right)-b\left(r-r^{\prime}\right)\right] f\left(r^{\prime}, \rho^{\prime}, t\right) \mathrm{d} \rho^{\prime} \mathrm{d} r^{\prime}
$$

and

$$
c(f)=\int_{\mathbb{R}^{2}} w\left(r-r^{\prime}\right)\left[\frac{z_{1}}{2}\left(b\left(\rho-\rho^{\prime}\right)+1\right)-\frac{z_{2}}{2}\left(b\left(r-r^{\prime}\right)-1\right)\right] f\left(r^{\prime}, \rho^{\prime}, t\right) \mathrm{d} \rho^{\prime} \mathrm{d} r^{\prime} .
$$

Krupp analysed the qualitative behaviour of solutions to (5). Due to the continuous increase in strength, the ratings increase in time. Therefore, an appropriately shifted problem was studied, in which the ratings converged exponentially fast to the intrinsic strength in the case $w=1$.

In this paper, we propose a more general approach to describe how a player's strength changes in encounters. We assume that individuals benefit from every game and increase their strength because of these interactions. However, the extent of the benefit depends on several factors - first, players with a lower rating benefit more. Second, the stronger the opponent, the more a win pushes the intrinsic strength. Furthermore, the individual performance changes due to small fluctuations, accounting for 
variations in the mental strength or personal fitness on a day. Based on the microscopic interaction laws, we derive the corresponding kinetic Boltzmann-type and limiting Fokker-Planck equations and analyse their behaviour. In the case of no diffusion, we can show that the strength and ratings of the appropriately shifted PDE converge, while we observe the formation of non-measure valued steady states in the case of diffusion. We illustrate our analytic results with numerical simulations of the kinetic as well as the limiting Fokker-Planck equation. The simulations give important insights into the dynamics, especially in situations where we are not able to prove rigorous results. The proposed interaction laws are a first step to develop and analyse more complicated rating models with dynamic strength. The next developments of the model should include losses in the player's strength to ensure that the strength stays within certain bounds.

The kinetic description of the Elo rating system allowed Junca and Jabin to analyse the qualitative behaviour of solutions. In the last decades, kinetic models have been used successfully to describe the behaviour of large multi-agent systems in socioeconomic applications. In all these applications, interactions among individuals are modelled as 'collisions', in which agents exchange goods (Delitala and Lorenzi 2014; Düring et al. 2017; Burger et al. 2013), wealth (Düring and Toscani 2007; Düring et al. 2008; Bellomo et al. 2013; Degond et al. 2014), opinion (Toscani 2006; Boudin et al. 2010; Düring et al. 2009; Motsch and Tadmor 2014; Albi et al. 2014; Düring and Wolfram 2015) or knowledge (Pareschi and Toscani 2014; Burger et al. 2016). For a general overview on interacting multi-agent systems and kinetic equations, we refer to the book of Pareschi and Toscani (2013).

This paper is organised as follows. We introduce a generalisation of the kinetic Elo model with variable intrinsic strength due to learning in Sect. 2. In Sect. 3, we derive the corresponding Fokker-Planck-type equation as the quasi-invariant limit of the Boltzmann-type model. Convergence towards steady states of a suitable shifted Fokker-Planck model is analysed in Sect. 4. We conclude by presenting various numerical simulations of the Boltzmann and the Fokker-Planck-type equation in Sect. 5.

\section{An Elo Model with Learning}

In this section, we introduce an Elo model, in which the rating and the intrinsic strength of the players change in time. The dynamics are driven by similar microscopic binary interactions as in the original model by Jabin and Junca (2015) and Krupp (2016). We state the specific microscopic interaction rules in each encounter and derive the corresponding limiting Fokker-Planck equation.

\subsection{Kinetic model}

We follow the notation introduced in Sect. 1 and denote the individual strength by $\rho$ and the rating by $R$. If two players with ratings $R_{i}$ and $R_{j}$ meet, their ratings and strength after the game are given by: 


$$
\begin{aligned}
R_{i}^{*} & =R_{i}+\gamma\left(S_{i j}-b\left(R_{i}-R_{j}\right)\right), \\
R_{j}^{*} & =R_{j}+\gamma\left(-S_{i j}-b\left(R_{j}-R_{i}\right)\right), \\
\rho_{i}^{*} & =\rho_{i}+\gamma h\left(\rho_{j}-\rho_{i}\right)+\eta, \\
\rho_{j}^{*} & =\rho_{j}+\gamma h\left(\rho_{i}-\rho_{j}\right)+\tilde{\eta} .
\end{aligned}
$$

The interaction rules are motivated by the following considerations: player ratings change with the outcome of each game [as in the original model (1) proposed by Jabin and Junca (2015)]. The random variable $S_{i j}$ corresponds to the score of the match and depends on the difference in strength of the two players. We assume that $S_{i j}$ takes the values \pm 1 with an expectation $\left\langle S_{i j}\right\rangle=b\left(\rho_{i}-\rho_{j}\right)$. Note that one could also assume that $S_{i j}$ is continuous, for example $S_{i j} \in[-1,+1]$. The constant parameter $\gamma>0$ controls the speed of adjustment.

The variables $\eta$ and $\tilde{\eta}$ are independent identically distributed random variables with mean zero and variance $\sigma^{2}$ which model small fluctuations due to day-linked performance in the mental strength or personal fitness.

The function $h$ describes the learning mechanism. We assume that $h$ takes the following form,

$$
h\left(\rho_{j}-\rho_{i}\right)=\left[\alpha h_{1}\left(\rho_{j}-\rho_{i}\right)+\beta h_{2}\left(\rho_{j}-\rho_{i}\right)\right] .
$$

The function $h_{1}$ corresponds to the increase in knowledge or skills because of interactions. We assume that each player learns in a game; however, players with a lower strength benefit more. A possible choice for $h_{1}$, which we shall use throughout this paper, is

$$
h_{1}\left(\rho_{j}-\rho_{i}\right)=1+b\left(\rho_{j}-\rho_{i}\right) \text {, }
$$

where $b$ is given by (2). Note that $b$ is an odd function. Since $h_{1}$ is positive, both players are able to learn and improve in each game, to an extent which depends on the difference in strengths, with a player with lower strength benefiting more.

The second function, $h_{2}$, models a change of strength due to gain or loss of selfconfidence due to winning or being defeated in a game. We assume that the loss of the stronger player is the same as the gain for the weaker one. Hence, we choose $h_{2}\left(\rho_{j}-\rho_{i}\right)=S_{i j} l\left(\rho_{j}-\rho_{i}\right)$ to be an odd, regular, bounded function which is vanishing at infinity, where the function $l$ corresponds to the net change of self-confidence. A possible choice which we adopt in the following corresponds to

$$
h_{2}\left(\rho_{j}-\rho_{i}\right)=S_{i j}\left[1-\tanh ^{2}\left(\rho_{j}-\rho_{i}\right)\right]
$$

Note that the expectation for the learning function is given by

$$
\begin{aligned}
& \left\langle h\left(\rho_{j}-\rho_{i}\right)\right\rangle=\left[\alpha h_{1}\left(\rho_{j}-\rho_{i}\right)+\beta\left\langle h_{2}\left(\rho_{j}-\rho_{i}\right)\right\rangle\right] \\
& \quad=\left[\alpha h_{1}\left(\rho_{j}-\rho_{i}\right)+\beta b\left(\rho_{i}-\rho_{j}\right)\left(1-\tanh ^{2}\left(\rho_{j}-\rho_{i}\right)\right)\right] .
\end{aligned}
$$


Fig. 1 Possible choices of $h_{1}$ and $h_{2}$

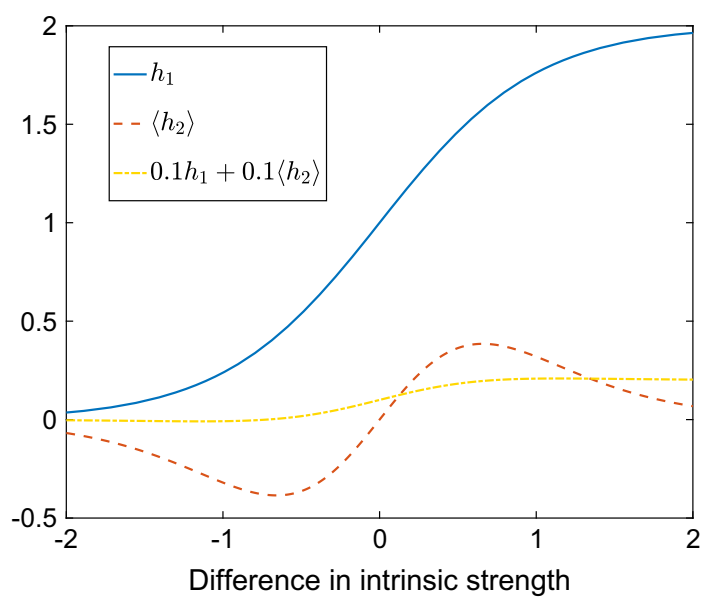

Figure 1 shows the function $h_{1},\left\langle h_{2}\right\rangle$ and $\langle h\rangle$ for the particular choice of $\alpha=\beta=0.1$ and $c=1$. If $\alpha>\beta$, players always improve in strength. In this case the strength and subsequently the rating will always increase in time. We see that, as in the original Elo model, the choices of interaction rules and the function $b(\cdot)$ preserve the total value of the rating pointwise and in mean, that is

$$
\left\langle R_{i}^{*}+R_{j}^{*}\right\rangle=R_{i}+R_{j}
$$

The evolution of the total strength depends on the choices of the function $h_{1}$ and $h_{2}$. Note that the function $h_{2}$ does not affect the total strength since

$$
\left\langle\rho_{j}^{*}+\rho_{j}^{*}\right\rangle-\left(\rho_{j}+\rho_{j}\right)=2 \gamma \alpha
$$

We see that that the proposed interaction rules result in a net increase of the total knowledge in every interactions. Therefore, we expect to see on overall increase in strength for all times.

The proposed interaction rules are a first step towards a more realistic modelling. Alternative learning mechanisms, such as the one proposed in the context of knowledge exchange in a large society (see Burger et al. (2016)) could be considered in the future. Here the individual with the lower knowledge level assumes the higher level after the interaction, while the stronger one did not gain anything in the encounter. Hence, the overall knowledge level is bounded by the maximum initial knowledge level for all times and the distribution of individuals converges to a Delta Dirac at that point. We expect a similar dynamics, if we were to apply that rule instead of (6). Developing learning mechanisms, which combine limitations of individual learning with the continuous evolution of the collective knowledge, will be an important aspect of future research developments.

Now we are able to state the evolution equation for the distribution of players $f_{\gamma}=f_{\gamma}(\rho, R, t)$ with respect to their rating $R$ and intrinsic strength $\rho$. For a fixed number of players, $N$, the interactions (6) induce a discrete-time Markov process with 
$N$-particle joint probability distribution $P_{N}\left(\rho_{1}, R_{1}, \rho_{2}, R_{2}, \ldots, \rho_{N}, R_{N}, \tau\right)$. One can write a kinetic equation for the one-marginal distribution function,

$$
P_{1}(\rho, R, \tau)=\int P_{N}\left(\rho, R, \rho_{2}, R_{2}, \ldots, \rho_{N}, R_{N}, \tau\right) \mathrm{d} \rho_{2} \mathrm{~d} R_{2} \cdots \mathrm{d} \rho_{N} \mathrm{~d} R_{N},
$$

using only one- and two-particle distribution functions (Cercignani 1988; Cercignani et al. 1994),

$$
\begin{aligned}
& P_{1}(\rho, R, \tau+1)-P_{1}(\rho, R, \tau)= \\
& \quad\left\langle\frac { 1 } { N } \left[\int P _ { 2 } ( \rho _ { i } , R _ { i } , \rho _ { j } , R _ { j } , \tau ) w ( R _ { i } - R _ { j } ) \left(\delta_{0}\left(\rho-\rho_{i}^{*}, R-R_{i}^{*}\right)\right.\right.\right. \\
& \left.\left.\left.\quad+\delta_{0}\left(\rho-\rho_{j}^{*}, R-R_{j}^{*}\right)\right) \mathrm{d} \rho_{i} \mathrm{~d} R_{i} \mathrm{~d} \rho_{j} \mathrm{~d} R_{j}-2 P_{1}(\rho, R, \tau)\right]\right\rangle .
\end{aligned}
$$

Here, $\langle\cdot\rangle$ denotes the mean operation with respect to the random variables $\eta, \tilde{\eta}$ and the function $w(\cdot)$ corresponds to the interaction rate function which depends on the difference of the ratings. This process can be continued to give a hierarchy of equations of so-called BBGKY-type (Cercignani 1988; Cercignani et al. 1994), describing the dynamics of the system of a large number of interacting agents. A standard approximation is to neglect correlations and assume the factorisation

$$
P_{2}\left(\rho_{i}, R_{i}, \rho_{j}, R_{j}, \tau\right)=P_{1}\left(\rho_{i}, R_{i}, \tau\right) P_{1}\left(\rho_{j}, R_{j}, \tau\right)
$$

By scaling time as $t=2 \tau / N$ and performing the thermodynamical limit $N \rightarrow \infty$, we can use standard methods of kinetic theory (Cercignani 1988; Cercignani et al. 1994) to show that the time-evolution of the one-agent distribution function $f_{\gamma}$ is governed by the following Boltzmann-type equation:

$$
\begin{aligned}
\frac{\mathrm{d}}{\mathrm{d} t} \int_{\Omega} \phi\left(\rho_{i}, R_{j}\right) f_{\gamma}\left(\rho_{i}, R_{i}, t\right) \mathrm{d} \rho_{i} \mathrm{~d} R_{i} \\
=\frac{1}{2}\left\langle\int_{\Omega} \int_{\Omega}\left(\phi\left(\rho_{i}^{*}, R_{j}^{*}\right)+\phi\left(\rho_{j}^{*}, R_{j}^{*}\right)-\phi\left(\rho_{i}, R_{i}\right)-\phi\left(\rho_{j}, R_{j}\right)\right)\right. \\
\left.\quad \times w\left(R_{i}-R_{j}\right) f_{\gamma}\left(\rho_{i}, R_{i}, t\right) f_{\gamma}\left(\rho_{j}, R_{j}, t\right) \mathrm{d} \rho_{j} \mathrm{~d} R_{j} \mathrm{~d} \rho_{i} \mathrm{~d} R_{i}\right\rangle
\end{aligned}
$$

where $\phi(\cdot)$ is a (smooth) test function, with support $\operatorname{supp}(\phi) \subseteq \Omega$. The function $w(\cdot)$ corresponds to the interaction rate function which depends on the difference of the ratings. If $w \equiv 1$ we consider a so-called all-play-all game. If $w$ has compact support only players with close ratings compete. Possible choices for $w$ are

$$
w\left(R_{i}-R_{j}\right)=e^{\frac{\log 2}{1+\left(R_{i}-R_{j}\right)^{2}}}-1 \text { or } w\left(R_{i}-R_{j}\right)=\chi_{\left\{\left|R_{i}-R_{j}\right| \leq c\right\}} .
$$

where $\chi$ denotes the indicator function (or smoothed variants thereof). 
In the following we shall analyse (11) as well as different asymptotic limits of it. The presented analysis is based on the following assumptions:

(A1) Let $\Omega=\mathbb{R}^{2}$ or a bounded Lipschitz domain $\Omega \subset \mathbb{R}^{2}$.

$(\mathcal{A} 2)$ Let $f_{0} \in H^{1}(\Omega)$ with $f_{0} \geq 0$ and compact support. Furthermore we assume that it has mean value zero, and bounded moments up to order two. Hence

$$
\int_{\Omega} f_{0}(\rho, R) \mathrm{d} \rho \mathrm{d} R=1, \int_{\Omega} R f_{0}(\rho, R) \mathrm{d} \rho \mathrm{d} R=0, \text { and } \int_{\Omega} \rho f_{0}(\rho, R) \mathrm{d} \rho \mathrm{d} R=0 .
$$

(A3) The random variables $\eta, \tilde{\eta}$ in (6) have the same distribution, zero mean, $\langle\eta\rangle=0$, and variance $\sigma_{\eta}^{2}$.

( $\mathcal{A} 4)$ Let the interaction rate function $w \geq 0$ be an even function with $w \in C^{2}(\Omega) \cap$ $L^{\infty}(\Omega)$.

The kinetic Elo model can be formulated on the whole space as well as on a bounded domain. In reality, the Elo ratings of top chess players vary between 2000 and 3000 , which provides evidence for the assumption of a bounded domain $\Omega$. However, sometimes it is easier to study the dynamics of models on the whole space, i.e. without boundary effects. We will generally work on the bounded domain, and clearly state where we deviate from this assumption, e.g. when we study the asymptotic behaviour of moments. The second assumption states the necessary regularity assumptions on the initial data, which we shall use in the analysis of the moments and the existence proof.

\subsection{Analysis of the moments}

We start by studying basic properties of the Boltzmann-type equation (11) such as mass conservation and the evolution of the first and second moments with respect to the strength and the ratings. Throughout this section we consider the problem in the whole space.

Conservation of Mass Setting $\phi\left(\rho_{i}, R_{i}\right)=1$ in Eq. (11) we see that

$$
\frac{\mathrm{d}}{\mathrm{d} t} \int_{\mathbb{R}^{2}} f_{\gamma}\left(\rho_{i}, \mathbb{R}, t\right) \mathrm{d} R \mathrm{~d} \rho=0
$$

Therefore, the total mass is conserved, that is

$$
\int_{\mathbb{R}^{2}} f_{\gamma}(R, \rho, t) \mathrm{d} R \mathrm{~d} \rho=1, \text { for all times } t \geq 0
$$

Moments with Respect to the Rating The $s$ th moment, for $s \in \mathbb{N}$, with respect to $R_{i}$ is defined as

$m_{R_{i}}(t)=\int_{\mathbb{R}^{2}} R_{i} f_{\gamma}\left(\rho_{i}, R_{i}, t\right) \mathrm{d} R_{i} \mathrm{~d} \rho_{i}$ and $\mu_{s, R_{i}}(t)=\int_{\mathbb{R}^{2}} R_{i}^{s} f_{\gamma}\left(\rho_{i}, R_{i}, t\right) \mathrm{d} R_{i} \mathrm{~d} \rho_{i}$, 
where $m_{R_{i}}=M_{1, R_{i}}$. We choose $\phi\left(\rho_{i}, R_{i}\right)=R_{i}$. Due to $(\mathcal{A} 2)$ and the symmetry of $b(\cdot)$ we obtain

$$
\begin{aligned}
\frac{\mathrm{d}}{\mathrm{d} t} m_{R_{i}(t)}= & \frac{1}{2} \gamma \int_{\mathbb{R}^{4}} f_{\gamma}\left(\rho_{i}, R_{i}, t\right) f_{\gamma}\left(\rho_{j}, R_{j}, t\right) \times \\
& \times\left(b\left(\rho_{i}-\rho_{j}\right)-b\left(R_{i}-R_{j}\right)\right. \\
& \left.+b\left(\rho_{j}-\rho_{i}\right)-b\left(R_{j}-R_{i}\right)\right) w\left(R_{i}-R_{j}\right) \mathrm{d} R_{j} \mathrm{~d} \rho_{j} \mathrm{~d} R_{i} \mathrm{~d} \rho_{i}=0 .
\end{aligned}
$$

Hence, the mean value w.r.t. the rating is preserved in time and therefore

$$
m_{R_{i}(t)}=0, \text { for all times } t \geq 0 \text {. }
$$

The evolution of the second moment can be obtained by setting $\phi\left(\rho_{i}, R_{i}\right)=R_{i}{ }^{2}$. We see that

$$
\begin{aligned}
\frac{\mathrm{d}}{\mathrm{d} t} & M_{2, R_{i}}(t) \\
= & \frac{1}{2} \int_{\mathbb{R}^{4}} f_{\gamma}\left(\rho_{i}, R_{i}, t\right) f_{\gamma}\left(\rho_{j}, R_{j}, t\right) w\left(R_{i}-R_{j}\right) \times \\
& \times\left[\gamma^{2}\left(\left(b\left(\rho_{i}-\rho_{j}\right)-b\left(R_{i}-R_{j}\right)\right)^{2}+\left(b\left(\rho_{j}-\rho_{i}\right)-b\left(R_{j}-R_{i}\right)\right)^{2}\right)\right. \\
& \left.+2 \gamma\left(R_{i}\left(b\left(\rho_{i}-\rho_{j}\right)-b\left(R_{i}-R_{j}\right)\right)+R_{j}\left(b\left(\rho_{j}-\rho_{i}\right)-b\left(R_{j}-R_{i}\right)\right)\right)\right] \mathrm{d} R_{j} \mathrm{~d} \rho_{j} \mathrm{~d} R_{i} \mathrm{~d} \rho_{i} .
\end{aligned}
$$

The second term in the integral is non-positive, and we obtain the bound

$$
\frac{\mathrm{d}}{\mathrm{d} t} M_{2, R_{i}}(t) \leq 4 \gamma^{2}\|b\|_{\infty}^{2}
$$

Hence, the second moment grows at most linearly and remains bounded for finite times. Note that the integral is negative for $\gamma$ small enough, which implies a decreasing second moment.

\section{Moments with Respect to the Strength}

The moments with respect to strength are defined in an analogous way, that is

$$
m_{\rho_{i}}(t)=\int_{\mathbb{R}^{2}} \rho_{i} f\left(\rho_{i}, R_{i}, t\right) d R_{i} d \rho_{i} \text { and } \mu_{s, \rho_{i}}(t)=\int_{\mathbb{R}^{2}} \rho_{i}{ }^{s} f\left(\rho_{i}, R_{i}, t\right) d R_{i} d \rho_{i},
$$

for $s \in \mathbb{N}$ and using again $m_{\rho_{i}}(t)=M_{1, \rho_{i}}$. Since $(\mathcal{A} 2)$ holds, we see that for $\phi\left(\rho_{i}, R_{i}\right)=\rho_{i}$, we have

$$
\begin{aligned}
\frac{\mathrm{d}}{\mathrm{d} t} m_{\rho_{i}}(t)= & \frac{1}{2} \gamma \int_{\mathbb{R}^{4}} f_{\gamma}\left(\rho_{i}, R_{i}, t\right) f_{\gamma}\left(\rho_{j}, R_{j}, t\right) w\left(R_{i}-R_{j}\right)\left[\left\langleh\left(\rho_{j}-\rho_{i}\right)\right.\right. \\
& \left.\left.+h\left(\rho_{i}-\rho_{j}\right)\right\rangle\right] \mathrm{d} \rho_{j} \mathrm{~d} R_{j} \mathrm{~d} \rho_{i} \mathrm{~d} R_{i} .
\end{aligned}
$$


Therefore,

$$
\begin{aligned}
& -\gamma\|\langle h\rangle\|_{\infty} \leq \frac{\mathrm{d}}{\mathrm{d} t} m_{\rho_{i}}(t) \\
& \quad \leq \frac{1}{2} \gamma \int_{\mathbb{R}^{4}} 2\|\langle h\rangle\|_{\infty} f_{\gamma}\left(\rho_{i}, R_{i}, t\right) f_{\gamma}\left(\rho_{j}, R_{j}, t\right) \mathrm{d} \rho_{j} \mathrm{~d} R_{j} \mathrm{~d} \rho_{i} \mathrm{~d} R_{i} \\
& \quad \leq \gamma\|\langle h\rangle\|_{\infty},
\end{aligned}
$$

which implies that the mean value is bounded for all times $t \in[0, T]$ and that $\left|m_{\rho_{i}}(t)\right|$ grows at most linearly in time if $h(\cdot)$ is bounded. If we consider the specific interaction rules (8)-(12), we obtain

$$
\frac{\mathrm{d}}{\mathrm{d} t} m_{\rho_{i}}(t)=\gamma \alpha \int_{\mathbb{R}^{4}} w\left(R_{i}-R_{j}\right) f_{\gamma}\left(\rho_{i}, R_{i}, t\right) f_{\gamma}\left(\rho_{j}, R_{j}, t\right) \mathrm{d} \rho_{j} \mathrm{~d} R_{j} \mathrm{~d} \rho_{i} \mathrm{~d} R_{i} \leq \gamma \alpha,
$$

with equality holding in the 'all-play-all' case $w=1$. The evolution of the second moment $M_{2, \rho_{i}}$ can be computed by setting $\phi\left(\rho_{i}, R_{i}\right)=\rho_{i}{ }^{2}$. We see that

$$
\begin{aligned}
\frac{\mathrm{d}}{\mathrm{d} t} M_{2, \rho_{i}}(t)= & \frac{1}{2} \int_{\mathbb{R}^{4}}\left(\gamma^{2}\left[\left\langle h\left(\rho_{j}-\rho_{i}\right)^{2}\right\rangle+\left\langle h\left(\rho_{i}-\rho_{j}\right)^{2}\right\rangle\right]\right. \\
& +2 \gamma\left[\rho_{i}\left\langle h\left(\rho_{j}-\rho_{i}\right)\right\rangle+\rho_{j}\left\langle h\left(\rho_{i}-\rho_{j}\right)\right\rangle\right] \\
& \left.+2 \sigma^{2}(\gamma)\right) w\left(R_{i}-R_{j}\right) f_{\gamma}\left(\rho_{i}, R_{i}, t\right) f_{\gamma}\left(\rho_{j}, R_{j}, t\right) \mathrm{d} \rho_{j} \mathrm{~d} R_{j} \mathrm{~d} \rho_{i} \mathrm{~d} R_{i} \\
\leq & \gamma^{2}\left\|\left\langle h^{2}\right\rangle\right\|_{\infty}+\sigma^{2}(\gamma)+4 \gamma\left|m_{\rho_{i}}(t)\right| .
\end{aligned}
$$

If $h(\cdot)$ is bounded, the second moment grows at most at polynomial rate. Since the second moment of $f_{0}$ is bounded (see assumption $(\mathcal{A} 2)$ ), it remains finite for all times $t \in[0, T]$.

\section{The Fokker-Planck Limit}

In the last section, we analysed the evolution of moments to the Boltzmann-type equation (11). However, it is often more useful to study the dynamics of simplified models (generally of Fokker-Planck type), which can be derived in particular asymptotic limits. These asymptotics provide a good approximation of the stationary profiles of the kinetic equation. In what follows, we consider the so-called quasi-invariant limit, in which diffusion and the outcome of the game influence the long-time dynamics. More specifically, we consider the limit

$$
\gamma \rightarrow 0, \sigma_{\eta} \rightarrow 0 \text { such that } \frac{\sigma_{\eta}^{2}}{\gamma}=: \sigma^{2} \text { is kept fixed. }
$$

In Appendix A, we derive the following Fokker-Planck limit: the differential form of (49) is given by (writing $t$ instead of $\tau$ ) 


$$
\begin{aligned}
\frac{\partial f(\rho, R, t)}{\partial t}= & -\frac{\partial}{\partial R}(a[f] f(\rho, R, t))-\frac{\partial}{\partial \rho}(c[f] f(\rho, R, t)) \\
& +\frac{\sigma^{2}}{2} d[f] \frac{\partial^{2}}{\partial \rho^{2}} f(\rho, R, t) \text { in } \Omega \times(0, T),
\end{aligned}
$$

where

$$
\begin{gathered}
\begin{array}{c}
a[f]=a[f](\rho, R, t)=\int_{\mathbb{R}^{2}} w\left(R-R_{j}\right)\left(b\left(\rho-\rho_{j}\right)-b\left(R-R_{j}\right)\right) \\
\times f\left(\rho_{j}, R_{j}, t\right) \mathrm{d} \rho_{j} \mathrm{~d} R_{j},
\end{array} \\
\begin{array}{r}
c[f]=c[f](\rho, R, t)=\int_{\mathbb{R}^{2}} w\left(R-R_{j}\right)\left(\alpha h_{1}\left(\rho_{j}-\rho\right)+\beta\left\langle h_{2}\left(\rho_{j}-\rho\right)\right\rangle\right) \\
\times f\left(\rho_{j}, R_{j}, t\right) \mathrm{d} \rho_{j} \mathrm{~d} R_{j},
\end{array} \\
\begin{array}{c}
d[f]=d[f](R, t)=\int_{\mathbb{R}^{2}} w\left(R-R_{j}\right) f\left(\rho_{j}, R_{j}, t\right) \mathrm{d} \rho_{j} \mathrm{~d} R_{j} .
\end{array}
\end{gathered}
$$

We consider Eq. (16) with initial datum $f_{0}$ satisfying assumption $(\mathcal{A} 2)$ in the following. Note that (16) includes the non-local operator $a[f]$, corresponding to the change of the ratings, similar as in the Fokker-Planck equations (3) and (5) obtained in Jabin and Junca (2015) and Krupp (2016), respectively. The non-local operator $c[f]$ in the transport terms corresponds to the change of the individual strengths, while the operator $d[f]$ describes the fluctuations of the individual strength due to encounters.

\subsection{Qualitative Properties of the Fokker-Planck Equation}

We continue by discussing qualitative properties of the Fokker-Planck equation (16). We shall see that several properties, which we observed for the Boltzmann-type equation (11), can be transferred.

Conservation of mass and positivity of solution: Due to mass conservation and ( $\mathcal{A} 2)$, we have that

$$
\int_{\mathbb{R}^{2}} f(\rho, R, t) \mathrm{d} \rho \mathrm{d} R=\int_{\mathbb{R}^{2}} f_{0}(\rho, R) \mathrm{d} \rho \mathrm{d} R=1 \text { for all } t \geq 0 .
$$

Using similar arguments as in Torregrossa and Toscani (2018), we can directly prove that the Fokker-Planck equation maintains the positivity of the solution. Let $v_{m}(t)=$ $\left(\rho_{m}(t), R_{m}(t)\right)$ denote the minimum, which is obtained at time $\tilde{t}$. Clearly, if at certain time $\tilde{t} \geq 0$ the function equals zero, i.e. $f(\rho, R, \tilde{t})=0$, this point is a stationary point or a local minimum, hence

$$
\frac{\partial}{\partial R} f\left(v_{m}, \tilde{t}\right)=0, \quad \frac{\partial}{\partial \rho} f\left(v_{m}, \tilde{t}\right)=0, \quad \frac{\partial^{2}}{\partial R^{2}} f\left(v_{m}, \tilde{t}\right) \geq 0, \quad \frac{\partial^{2}}{\partial \rho^{2}} f\left(v_{m}, \tilde{t}\right) \geq 0 .
$$


Evaluating (16) in $\left(v_{m}, \tilde{t}\right)$ gives

$$
\begin{aligned}
\frac{\partial}{\partial t} f\left(v_{m}, \tilde{t}\right)= & f\left(v_{m}, \tilde{t}\right)\left(-\frac{\partial}{\partial R} a[f]\left(v_{m}, \tilde{t}\right)-\frac{\partial}{\partial \rho} c[f]\left(v_{m}, \tilde{t}\right)\right) \\
& -a[f]\left(v_{m}, \tilde{t}\right) \frac{\partial}{\partial R} f\left(v_{m}, \tilde{t}\right)-c[f]\left(v_{m}, \tilde{t}\right) \frac{\partial}{\partial r} f\left(v_{m}, \tilde{t}\right) \\
& +\frac{\sigma^{2}}{2}\left(v_{m}, \tilde{t}\right) d[f] \frac{\partial^{2}}{\partial \rho^{2}}\left(f\left(v_{m}, \tilde{t}\right)\right) \geq 0,
\end{aligned}
$$

which implies that the function $f$ is non-decreasing in time and cannot assume negative values.

Evolution of the moments We now consider the evolution of the moments of the solution of (16) using the interaction rules (8) and (9). Similar calculations as in Sect. 2.2 confirm the expected behaviour-due to the continuous increase in strength in each game the system does not converge to a steady state and therefore the respective mean of the solution is non-decreasing in time. Summarising the results, we have

$$
\begin{aligned}
\frac{\partial}{\partial t} \int_{\mathbb{R}^{2}} R f(\rho, R, t) \mathrm{d} R \mathrm{~d} \rho & =0 \\
\frac{\partial}{\partial t} \int_{\mathbb{R}^{2}} \rho f(\rho, R, t) \mathrm{d} R \mathrm{~d} \rho & =\alpha \int_{\mathbb{R}^{2}} c[f] f(\rho, R, t) \mathrm{d} R \mathrm{~d} \rho \\
& =\alpha \int_{\mathbb{R}^{4}} w\left(R-R_{j}\right) f(\rho, R, t) f\left(\rho_{j}, R_{j}, t\right) \mathrm{d} \rho_{j} \mathrm{~d} R_{j} \mathrm{~d} \rho \mathrm{d} R .
\end{aligned}
$$

The previous results confirm that due to the continuous increase in strength in each game, rating and skills tend to become increasingly distant from each other. Therefore, we adopt an idea by Krupp (2016) and study the evolution of a suitably shifted problem instead. We define

$$
g(\rho, R, t)=f(\rho+H(\rho, R, t), R, t)
$$

where the scaling function $H$ is given by

$$
\frac{\partial H(\rho, R, t)}{\partial t}=\int_{\mathbb{R}^{2}} \alpha w\left(R-R_{j}\right) f\left(\rho_{j}, R_{j}, t\right) \mathrm{d} \rho_{j} \mathrm{~d} R_{j}=\alpha d[f] .
$$

This scaling ensures that the mean value is preserved in time. The corresponding evolution equation for $g(\rho, R, t)$ is given by

$$
\frac{\partial g(\rho, R, t)}{\partial t}=-\frac{\partial}{\partial R}(a[g] g(\rho, R, t))-\frac{\partial}{\partial \rho}(\tilde{c}[g] g(\rho, R, t))+\frac{\sigma^{2}}{2} d[g] \frac{\partial^{2}}{\partial \rho^{2}} g(\rho, R, t),
$$

where 


$$
\begin{aligned}
\tilde{c}[g]= & \tilde{c}[g](\rho, R, t) \\
& =\int_{\mathbb{R}^{2}}\left(\alpha b\left(\rho_{j}-\rho\right)+\beta\left\langle h_{2}\left(\rho_{j}-\rho\right)\right\rangle\right) w\left(R-R_{j}\right) g\left(\rho_{j}, R_{j}, t\right) \mathrm{d} \rho_{j} \mathrm{~d} R_{j} .
\end{aligned}
$$

Now, the mean value of $g(\rho, R, t)$ is constant w.r.t. both $R$ and $\rho$ and we can normalise

$$
\int_{\mathbb{R}^{2}} R g(\rho, R, t) \mathrm{d} \rho \mathrm{d} R=0 \text {, and } \int_{\mathbb{R}^{2}} \rho g(\rho, R, t) \mathrm{d} \rho \mathrm{d} R=0 .
$$

In a general setting, it is not possible to compute scaling function explicitly. However, in 'all-meet-all' tournaments, that is $w\left(R-R_{j}\right)=1$, and in case of the specific interaction rules (8)-(9), we obtain that

$$
H(\rho, R, t)=\alpha t .
$$

Therefore, in the rest of this paper, we consider the following problem on a bounded domain $\Omega \subset \mathbb{R}^{2}$, with no-flux boundary condition

$$
\begin{aligned}
\frac{\partial g(\rho, R, t)}{\partial t}= & -\frac{\partial}{\partial R}(a[g] g(\rho, R, t))-\frac{\partial}{\partial \rho}(\tilde{c}[g] g(\rho, R, t)) \\
& +\frac{\sigma^{2}}{2} d[g] \frac{\partial^{2}}{\partial \rho^{2}} g(\rho, R, t) \quad \text { in } \Omega \times(0, T), \\
\frac{\partial}{\partial \nu} g & =0 \quad \text { on } \partial \Omega, \\
g(\rho, R, 0) & =g_{0}(\rho, R) \text { in } \Omega .
\end{aligned}
$$

Here, $v$ denotes the unit outer normal vector. Note that the existence of solutions to (21a) on the whole domain is more involved, since we would need to prove that the solution decays sufficiently as $R$ and $\rho$ tend to infinity. Therefore, we consider the equation on a bounded domain only.

\subsection{Analysis of the Fokker-Planck Equation}

In the section, we prove existence of weak solutions to (21). The main result reads as follows.

Theorem 1 Let $(\mathcal{A} 1)$ be satisfied, $g_{0} \in H^{1}(\Omega)$ and $0 \leq g_{0} \leq M_{0}$ for some $M_{0}>0$ and assume $h_{1},\left\langle h_{2}\right\rangle, b \in L^{\infty}(\Omega) \cap C^{2}(\Omega)$. Then, there exists a weak solution $g \in$ $L^{2}\left(0, T ; H^{1}(\Omega)\right) \cap H^{1}\left(0, T ; H^{-1}(\Omega)\right)$ to (21a)-(21c), satisfying $0 \leq g \leq M_{0} e^{\lambda t}$ for all $(\rho, R) \in \Omega, t>0$, with a constant $\lambda>0$ depending on the functions $h_{1},\left\langle h_{2}\right\rangle, b$ and $w$.

The presented existence proof was adapted from a similar argument for a nonlinear Fokker-Planck equation describing the dynamics of agents in an economic market (see Düring et al. (2017)). However, Eq. (21a) has an additional nonlinearity in the derivative w.r.t. the rating $R$. We divide the proof in several steps for the ease of 
presentation. In Step 0, we regularise the nonlinear Fokker-Planck equation (21a) by adding a Laplace operator with small diffusivity $\mu \geq 0$. We linearise the equation in Step 1 and show existence of a unique solution for this problem. In Step 2, we derive the necessary $L^{\infty}$ estimates to use Leray-Schauder's fixed-point theorem and show existence of solutions to the nonlinear regularised problem. In Step 3, we present additional $H^{1}$ estimates, which allow us to pass to the limit $\mu \rightarrow 0$ in Step 4 .

Proof Step 0: the regularised problem. For $M>0$, let us denote by $g_{M}=$ $\max \{0, \min \{g, M\}\}$ and define

$$
\begin{aligned}
K_{M}[g] & =\int_{\Omega}\left[\alpha h_{1}\left(\rho_{j}-\rho\right)+\beta\left\langle h_{2}\left(\rho_{j}-\rho\right)\right\rangle\right] w\left(R-R_{j}\right) g_{M}\left(\rho_{j}, R_{j}, t\right) \mathrm{d} \rho_{j} \mathrm{~d} R_{j}, \\
L_{M}[g] & =\int_{\Omega}\left[b\left(\rho-\rho_{j}\right)-b\left(R-R_{j}\right)\right] w\left(R-R_{j}\right) g_{M}\left(\rho_{j}, R_{j}\right) \mathrm{d} \rho_{j} \mathrm{~d} R_{j} .
\end{aligned}
$$

Next we consider the regularised nonlinear problem for $0<\mu<1$,

$$
\begin{aligned}
\frac{\partial}{\partial t} g_{\mu}= & -\frac{\partial}{\partial R}\left(L_{M}\left[g_{\mu}\right] g_{\mu}(\rho, R, t)\right)-\frac{\partial}{\partial \rho}\left(K_{M}\left[g_{\mu}\right] g_{\mu}(\rho, R, t)\right) \\
& +\frac{\sigma^{2}}{2} d\left[g_{\mu}\right] \frac{\partial^{2}}{\partial \rho^{2}}\left(g_{\mu}(\rho, R, t)\right)+\mu \Delta\left(g_{\mu}(\rho, R, t)\right) \text { in } \Omega \times(0, T),
\end{aligned}
$$

with boundary and initial conditions given by

$$
\frac{\partial}{\partial \nu} g_{\mu}=0 \text { on } \partial \Omega, \text { and } g_{\mu}(\rho, R, 0)=g_{0} \text { on } \Omega
$$

The weak formulation of (22) is given by

$$
\begin{aligned}
\int_{0}^{T}\left\langle\frac{\partial}{\partial t} g_{\mu}, v\right\rangle \mathrm{d} t= & \int_{0}^{T} \int_{\Omega}\left(L_{M}\left[g_{\mu}\right] g_{\mu} \frac{\partial}{\partial R} v\right. \\
& \left.+K_{M}\left[g_{\mu}\right] g_{\mu} \frac{\partial}{\partial \rho} v-\frac{\sigma^{2}}{2} d\left[g_{\mu}\right] \frac{\partial}{\partial \rho} g_{\mu} \frac{\partial}{\partial \rho} v-\mu \frac{\partial}{\partial R} g_{\mu} \frac{\partial}{\partial R} v\right) \mathrm{d} R \mathrm{~d} \rho \mathrm{d} t,
\end{aligned}
$$

where $\langle\cdot, \cdot\rangle$ is the dual product between $H^{1}(\Omega)$ and $H^{-1}(\Omega)$ and $v \in H^{1}(\Omega)$.

Step 1: solution of the linearised regularised problem. Next we want to apply Leray-Schauder's fixed-point theorem. Let $\tilde{g} \in L^{2}\left(0, T ; L^{2}(\Omega)\right), \theta \in[0,1]$ and $g^{+}=\max (g, 0)$. We introduce the operators $A: H^{1}(\Omega) \times H^{1}(\Omega) \rightarrow \mathbb{R}$ and $F: H^{1}(\Omega) \rightarrow \mathbb{R}:$

$$
\begin{aligned}
A\left(g_{\mu}, v\right) & =\int_{\Omega} \mu\left(\frac{\partial}{\partial R} g_{\mu} \frac{\partial}{\partial R} v+\frac{\partial}{\partial \rho} g_{\mu} \frac{\partial}{\partial \rho} v\right) \mathrm{d} R \mathrm{~d} \rho \\
F(v) & =\theta \int_{\Omega}\left(L_{M}[\tilde{g}] \tilde{g}^{+} \frac{\partial}{\partial R} v+K_{M}[\tilde{g}] \tilde{g}^{+} \frac{\partial}{\partial \rho} v-\frac{\sigma^{2}}{2} d[\tilde{g}] \frac{\partial}{\partial \rho} \tilde{g}^{+} \frac{\partial}{\partial \rho} v\right) \mathrm{d} R \mathrm{~d} \rho .
\end{aligned}
$$


The operator $A(\cdot, \cdot)$ is bilinear and continuous on $H^{1}(\Omega) \times H^{1}(\Omega)$. The quantities $\left|K_{M}[\tilde{g}]\right|$ and $\left|L_{M}[\tilde{g}]\right|$ are bounded (because of the assumption made on $h_{1},\left\langle h_{2}\right\rangle$ and $b$ ); therefore, $F$ is continuous in $H^{1}(\Omega)$. Because of Poincaré's inequality, for some constant $C_{1}$ and $C_{2}$

$$
A\left(g_{\mu}, g_{\mu}\right)=\mu \int_{\Omega}\left(\left|\frac{\partial}{\partial \rho} g_{\mu}\right|^{2}+\left|\frac{\partial}{\partial R} g_{\mu}\right|^{2}\right) \mathrm{d} R \mathrm{~d} \rho \geq C_{1} \mu\left\|g_{\mu}\right\|_{H^{1}(\Omega)}-C_{2}\left\|g_{\mu}\right\|_{2} .
$$

By corollary 23.26 in Zeidler (1990), there exists a unique solution $g_{\mu} \in$ $L^{2}\left(0, T ; H^{1}(\Omega)\right) \cap H^{1}\left(0, T ; H^{-1}(\Omega)\right)$ to

$$
\left\langle\frac{\partial}{\partial t} g_{\mu}, v\right\rangle+A\left(g_{\mu}, v\right)=F(v), t>0, g_{\mu}(0)=\theta g_{0} .
$$

This defines the fixed-point operator $V: L^{2}\left(0, T ; L^{2}(\Omega)\right) \times[0,1] \rightarrow L^{2}\left(0, T ; L^{2}(\Omega)\right)$, $(\tilde{g}, \theta) \mapsto V(\tilde{g}, \theta)=g_{\mu}$, where $g_{\mu}$ solves (26). This operator satisfies $V(\tilde{g}, 0)=$ 0 . Standard arguments, including Galerkin's method and estimates on $\left\|\frac{\partial}{\partial t} g_{\mu}\right\|_{L^{2}\left(0, T ; H^{-1}(\Omega)\right)}$, show that the operator $V$ is continuous (with constants depending on the regularisation parameter $\mu$ ). The operator is also compact, because $L^{2}\left(0, T ; H^{1}(\Omega)\right) \cap H^{1}\left(0, T ; H^{-1}(\Omega)\right)$ is compactly embedded in $L^{2}\left(0, T ; L^{2}(\Omega)\right)$ (see Simon (1986)). In order to apply the fixed-point theorem of Leray-Schauder, we need to show uniform estimates.

Step 2: uniform $L^{\infty}$ bound and existence of a fixed point. We start by proving upper and lower bounds for the function $g_{\mu}$. Let $g_{\mu}$ be a fixed point of $V(\cdot, \theta)$, i.e. $g_{\mu}$ solves (26) with $\tilde{g}=g_{\mu}$, and $\theta \in[0,1]$.

For a lower bound, choosing $v=g_{\mu}^{-}=\min \left\{0, g_{\mu}\right\} \in L^{2}\left(0, T ; H^{1}(\Omega)\right)$ as test function in (26) and integrating in time, we obtain

$$
\frac{\mathrm{d}}{\mathrm{d} t}\left\|g_{\mu}^{-}\right\|_{L^{2}(\Omega)}^{2}=-2 A\left(g_{\mu}, g_{\mu}^{-}\right) \leq-C_{1}\left\|g_{\mu}^{-}\right\|_{2}^{2} \leq 0
$$

This shows that if $g_{\mu}(0)^{-}=0$, then $g_{\mu}(t)^{-}=0$ for all $t>0$. Hence, in all previous computations and in (24)-(25), we can replace $g_{\mu}^{+}$with $g_{\mu}$.

Now we show an upper bound. Let $g_{*}=\left(g_{\mu}-M\right)^{+}$, where $M=M_{0} e^{\lambda t}$, for some $\lambda>0$ to be determined below. We choose $v=g_{*} \in L^{2}\left(0, T ; H^{1}(\Omega)\right)$ as test function in (23). By assumption, $g_{0} \leq M_{0}$, i.e. $g_{*}(0)=\left(g_{0}-M_{0}\right)^{+}=0$. We note that $\frac{\partial}{\partial t} M=\lambda M$ and $\frac{1}{2} \frac{\partial}{\partial \rho}\left(g_{*}^{2}\right)=\left(g_{\mu}-M\right) \frac{\partial}{\partial \rho} g_{*}$. Then,

$$
\begin{aligned}
\frac{1}{2} \int_{\Omega} g_{*}(t)^{2} \mathrm{~d} R \mathrm{~d} \rho= & \int_{0}^{t}\left[-\lambda \int_{\Omega} M g_{*} \mathrm{~d} R \mathrm{~d} \rho-A\left(g_{\mu}, g_{*}\right)+F\left(g_{*}\right)\right] \mathrm{d} s \\
= & \int_{0}^{t} \frac{\sigma^{2}}{2} \int_{\Omega} d\left[g_{\mu}\right] \frac{\partial}{\partial \rho}\left(\left(g_{\mu}-M\right)+M\right) \frac{\partial}{\partial \rho} g_{*} \mathrm{~d} R \mathrm{~d} \rho \\
& -\mu \int_{\Omega}\left|\nabla g_{*}\right|^{2} \mathrm{~d} R \mathrm{~d} \rho+\theta(I+J) \mathrm{d} s
\end{aligned}
$$




$$
\leq \int_{0}^{t} \theta(I+J) \mathrm{d} s
$$

where $I=\int_{\Omega} L_{M}\left[g_{\mu}\right] g_{\mu} \frac{\partial}{\partial R} g_{*} \mathrm{~d} R \mathrm{~d} \rho$ and $J=\int_{\Omega} K_{M}\left[g_{\mu}\right] g_{\mu} \frac{\partial}{\partial \rho} g_{*} \mathrm{~d} R \mathrm{~d} \rho$. Let us consider $I$ and $J$, separately:

$$
\begin{aligned}
I & =\int_{\Omega} L_{M}\left[g_{\mu}\right]\left(g_{\mu}-M\right) \frac{\partial}{\partial R} g_{*} \mathrm{~d} R \mathrm{~d} \rho+\int_{\Omega} L_{M}\left[g_{\mu}\right] M \frac{\partial}{\partial R} g_{*} \mathrm{~d} R \mathrm{~d} \rho \\
& =-\frac{1}{2} \int_{\Omega} \frac{\partial}{\partial R}\left[L_{M}\left[g_{\mu}\right]\right] g_{*}^{2} \mathrm{~d} R \mathrm{~d} \rho-\int_{\Omega} \frac{\partial}{\partial R}\left[L_{M}\left[g_{\mu}\right]\right] M g_{*} \mathrm{~d} R \mathrm{~d} \rho \\
J & =\int_{\Omega} K_{M}\left[g_{\mu}\right]\left(g_{\mu}-M\right) \frac{\partial}{\partial \rho} g_{*} \mathrm{~d} R \mathrm{~d} \rho+\int_{\Omega} L_{M}\left[g_{\mu}\right] M \frac{\partial}{\partial \rho} g_{*} \mathrm{~d} R \mathrm{~d} \rho \\
& =-\frac{1}{2} \int_{\Omega} \frac{\partial}{\partial \rho}\left[K_{M}\left[g_{\mu}\right]\right] g_{*}^{2} \mathrm{~d} R \mathrm{~d} \rho-\int_{\Omega} \frac{\partial}{\partial \rho}\left[K_{M}\left[g_{\mu}\right]\right] M g_{*} \mathrm{~d} R \mathrm{~d} \rho .
\end{aligned}
$$

The assumptions on $h_{1},\left\langle h_{2}\right\rangle$ and $b$ ensure that $\frac{\partial}{\partial R}\left[L_{M}\left[g_{\mu}\right]\right]$ and $\frac{\partial}{\partial \rho}\left[K_{M}\left[g_{\mu}\right]\right]$ are bounded. Hence,

$$
\begin{aligned}
\frac{1}{2} \int_{\Omega} g_{*}^{2} \mathrm{~d} R \mathrm{~d} \rho= & \int_{\Omega}\left(\frac{\partial}{\partial t} g_{*}\right) g_{*} \mathrm{~d} R \mathrm{~d} \rho \\
\leq & C\left(L_{M}\left[g_{\mu}\right], K_{M}\left[g_{\mu}\right]\right) \int_{\Omega} g_{*}^{2} \mathrm{~d} R \mathrm{~d} \rho \\
& +\left(C\left(L_{M}\left[g_{\mu}\right], K_{M}\left[g_{\mu}\right]\right)-\lambda\right) \int_{\Omega} M g_{*} \mathrm{~d} R \mathrm{~d} \rho .
\end{aligned}
$$

Choosing $\lambda$ large enough and using Gronwall's lemma, we obtain

$$
\int_{\Omega} g_{*}(t)^{2} \mathrm{~d} R \mathrm{~d} \rho \leq \int_{\Omega} g_{*}(0)^{2} \exp \left[2 C\left(L_{M}\left[g_{\mu}\right], K_{M}\left[g_{\mu}\right]\right) t\right] \mathrm{d} R \mathrm{~d} \rho=0 .
$$

Therefore, $g_{*}(t)=0$ for all $t>0$, which implies $g_{\mu}(t) \leq M$ for all $t>0$. This allows us to replace $L_{M}\left[g_{\mu}\right]$ with $a\left[g_{\mu}\right]$ and $K_{M}\left[g_{\mu}\right]$ with $\tilde{c}\left[g_{\mu}\right]$ in (23). The uniform $L^{\infty}$ bound provides the necessary bound for the fixed-point operator in $L^{2}\left(0, T ; L^{2}(\Omega)\right)$. This implies existence of a weak solution to (23).

Step 3: uniform $H^{1}$ bound. Our aim is to derive an $H^{1}$ bound which is independent of $\mu$. Choosing $v=g_{\mu}$ in (23) with $t$ instead of $T$, we obtain

$$
\begin{aligned}
\frac{1}{2} & \frac{\mathrm{d}}{\mathrm{d} t} \int_{\Omega} g_{\mu}(t)^{2} \mathrm{~d} R \mathrm{~d} \rho=\int_{\Omega} a\left[g_{\mu}\right] g_{\mu} \frac{\partial}{\partial R} g_{\mu} \mathrm{d} R \mathrm{~d} \rho+\int_{\Omega} \tilde{c}\left[g_{\mu}\right] g_{\mu} \frac{\partial}{\partial \rho} g \mathrm{~d} R \mathrm{~d} \rho \\
& -\int_{\Omega}\left(\frac{\sigma^{2}}{2} d\left[g_{\mu}\right]+\mu\right)\left|\frac{\partial}{\partial \rho} g_{\mu}\right|^{2} \mathrm{~d} R \mathrm{~d} \rho-\mu \int_{\Omega}\left|\frac{\partial}{\partial R} g_{\mu}\right|^{2} \mathrm{~d} R \mathrm{~d} \rho \\
= & -\frac{1}{2} \int_{\Omega} \frac{\partial}{\partial R} a\left[g_{\mu}\right] g_{\mu}^{2} \mathrm{~d} R \mathrm{~d} \rho-\frac{1}{2} \int_{\Omega} \frac{\partial}{\partial \rho} \tilde{c}\left[g_{\mu}\right] g_{\mu}^{2} \mathrm{~d} R \mathrm{~d} \rho \\
& -\int_{\Omega}\left(\frac{\sigma^{2}}{2} d\left[g_{\mu}\right]+\mu\right)\left|\frac{\partial}{\partial \rho} g_{\mu}\right|^{2} \mathrm{~d} R \mathrm{~d} \rho
\end{aligned}
$$




$$
-\mu \int_{\Omega}\left|\frac{\partial}{\partial R} g_{\mu}\right|^{2} \mathrm{~d} R \mathrm{~d} \rho .
$$

Because of the assumptions on $h_{1},\left\langle h_{2}\right\rangle$ and $b$ we have that $\left|-\frac{1}{2}\left(\frac{\partial}{\partial R} a\left[g_{\mu}\right]+\frac{\partial}{\partial \rho} \tilde{c}\left[g_{\mu}\right]\right)\right|<C$. Therefore, we can rewrite the above estimate as

$$
\begin{aligned}
& \frac{1}{2} \int_{\Omega} g_{\mu}(t)^{2} \mathrm{~d} R \mathrm{~d} \rho \\
& \quad+\int_{0}^{t}\left[\int_{\Omega}\left(\frac{\sigma^{2}}{2} d\left[g_{\mu}\right]+\mu\right)\left|\frac{\partial}{\partial \rho} g_{\mu}\right|^{2} \mathrm{~d} R \mathrm{~d} \rho+\mu \int_{\Omega}\left|\frac{\partial}{\partial R} g_{\mu}\right|^{2} \mathrm{~d} R \mathrm{~d} \rho\right] \mathrm{d} s \\
& \leq C \int_{0}^{t} \int_{\Omega} g_{\mu}(t)^{2} \mathrm{~d} R \mathrm{~d} \rho \mathrm{d} t+\frac{1}{2} \int_{\Omega} g(0)^{2} \mathrm{~d} R \mathrm{~d} \rho .
\end{aligned}
$$

Using Gronwall's lemma, the previous estimate guarantees (independent by $\mu$ ) estimates for $g_{\mu}(t)$, i.e.

$$
\left\|g_{\mu}\right\|_{L^{\infty}\left(0, T ; L^{2}(\Omega)\right)} \leq C .
$$

However, this does not ensure an (independent of $\mu$ ) estimate for $\frac{\partial}{\partial R} g_{\mu}$ and $\frac{\partial}{\partial \rho} g_{\mu}$. In order to obtain it, we differentiate (22a) with respect to $R$ and $\rho$ in the sense of distributions. This gives us estimates for $y:=\frac{\partial}{\partial R} g_{\mu}$ and $z:=\frac{\partial}{\partial \rho} g_{\mu}$. We obtain

$$
\begin{aligned}
\frac{\partial}{\partial t} y= & -\frac{\partial}{\partial R}\left(d\left[g_{\mu}\right] g_{\mu}+a\left[g_{\mu}\right] y\right)-\frac{\partial}{\partial \rho}\left(\tilde{c}\left[g_{\mu}\right] y\right)+\frac{\sigma^{2}}{2} \frac{\partial^{2}}{\partial \rho^{2}} y+\gamma \frac{\partial^{2}}{\partial R^{2}} y \\
& \text { in } \Omega \times(0, T) .
\end{aligned}
$$

Due to no-flux boundary condition (21b), Eq. (28) is complemented with

$$
\frac{\partial}{\partial \nu_{R}} y(\rho, R, t)=0 \quad \text { on } \partial \Omega,
$$

where $v_{R}$ is the component w.r.t. variable $R$ of the normal vector $v$ to $\Omega$. Furthermore, $y(\rho, R, 0)=\frac{\partial}{\partial R} g_{0}(\rho, R)$. Choosing $v \in L^{2}\left(0, T ; H_{0}^{1}(\Omega)\right)$ and setting $d^{\prime}\left[g_{\mu}\right]=\frac{\partial}{\partial R} d\left[g_{\mu}\right], \tilde{c}_{R}\left[g_{\mu}\right]=\frac{\partial}{\partial R} \tilde{c}\left[g_{\mu}\right]$ and $a_{R}\left[g_{\mu}\right]=\frac{\partial}{\partial R} a\left[g_{\mu}\right]$, we obtain the weak formulation of Eq. (28):

$$
\begin{aligned}
\int_{0}^{T}\left\langle\frac{\partial}{\partial t} y, v\right\rangle \mathrm{d} s= & \int_{0}^{T} \int_{\Omega}\left(a_{R}\left[g_{\mu}\right] g_{\mu} \frac{\partial}{\partial R} v+a\left[g_{\mu}\right] y \frac{\partial}{\partial R} v+\tilde{c}_{R}\left[g_{\mu}\right] g_{\mu} \frac{\partial}{\partial \rho} v\right. \\
& +\tilde{c}\left[g_{\mu}\right] y \frac{\partial}{\partial \rho} v-\frac{\sigma^{2}}{2} \frac{\partial}{\partial \rho}\left(d^{\prime}\left[g_{\mu}\right] g_{\mu}+d\left[g_{\mu}\right] y\right) \frac{\partial}{\partial \rho} v \\
& \left.-\mu\left(\frac{\partial}{\partial \rho} y \frac{\partial}{\partial \rho} v+\frac{\partial}{\partial R} y \frac{\partial}{\partial R} v\right)\right) \mathrm{d} R \mathrm{~d} \rho \mathrm{d} s .
\end{aligned}
$$


We introduce the operators

$$
\begin{aligned}
B_{y}(y, v)= & \int_{\Omega}-a\left[g_{\mu}\right] y \frac{\partial}{\partial R} v-\tilde{c}\left[g_{\mu}\right] y \frac{\partial}{\partial \rho} v+\frac{\sigma^{2}}{2} d\left[g_{\mu}\right] \frac{\partial}{\partial \rho} y \frac{\partial}{\partial \rho} v \\
& +\mu\left(\frac{\partial}{\partial \rho} y \frac{\partial}{\partial \rho} v+\frac{\partial}{\partial R} y \frac{\partial}{\partial R} v\right) \mathrm{d} R \mathrm{~d} \rho \\
G_{y}(v)= & \int_{\Omega} \tilde{c}_{R}\left[g_{\mu}\right] g_{\mu} \frac{\partial}{\partial \rho} v+a_{R}\left[g_{\mu}\right] g_{\mu} \frac{\partial}{\partial R} v \\
& -\frac{\sigma^{2}}{2} d^{\prime}\left[g_{\mu}\right] \frac{\partial}{\partial \rho} g_{\mu} \frac{\partial}{\partial \rho} v \mathrm{~d} R \mathrm{~d} \rho .
\end{aligned}
$$

Both operators $B_{y}: L^{2}\left(0, T ; H_{0}^{1}(\Omega)\right) \times L^{2}\left(0, T ; H_{0}^{1}(\Omega)\right) \rightarrow \mathbb{R}$ and $G_{y}:$ $L^{2}\left(0, T ; H_{0}^{1}(\Omega)\right) \rightarrow \mathbb{R}$ are linear and continuous. Garding's inequality implies

$$
\begin{aligned}
B_{y}(y, y)= & \int_{\Omega} \mu|\nabla y|^{2} \mathrm{~d} R \mathrm{~d} \rho+\frac{1}{2} \int_{\Omega}\left(\tilde{c}_{R}\left[g_{\mu}\right]+a_{R}\left[g_{\mu}\right]\right) y^{2} \mathrm{~d} \rho \mathrm{d} R \\
& +\frac{\sigma^{2}}{2} \int_{\Omega} d\left[g_{\mu}\right]\left|\frac{\partial}{\partial \rho} y\right|^{2} \mathrm{~d} R \mathrm{~d} \rho \\
\geq & \mu\|y\|_{H^{1}(\Omega)}^{2}-\left(\mu+\frac{1}{2}\left\|a\left[g_{\mu}\right]\right\|_{\infty}+\frac{1}{2}\left\|\tilde{c}\left[g_{\mu}\right]\right\|_{\infty}\right)\|y\|_{2}^{2} .
\end{aligned}
$$

Then, corollary 23.26 in Zeidler (1990) gives existence of a unique solution $y \in$ $L^{2}\left(0, T ; H_{0}^{1}(\Omega)\right) \cap H^{1}\left(0, T ; H^{-1}(\Omega)\right)$ to

$$
\left\langle\frac{\partial}{\partial t} y, v\right\rangle+B_{y}(y, v)=G_{y}(v), t>0, y(0)=y_{0}
$$

Choosing $v=y$ in (29), we obtain (using Young's and Garding's inequality)

$$
\begin{aligned}
& \frac{1}{2} \frac{\mathrm{d}}{\mathrm{d} t} \int_{\Omega} y(t)^{2} \mathrm{~d} R \mathrm{~d} \rho=-B_{y}(y, y)+G_{y}(y) \\
& \leq-\mu\|y\|_{H^{1}(\Omega)}^{2}+C\|y\|_{2}^{2} \\
& \quad+\frac{1}{2}\left(\left\|\frac{\partial^{2}}{\partial R^{2}} a\left[g_{\mu}\right]\right\|_{\infty}+\left\|\frac{\partial}{\partial \rho}\left(\frac{\partial}{\partial R} \tilde{c}\left[g_{\mu}\right]\right)\right\|_{\infty}\right) \int_{\Omega} g_{\mu}^{2}+y^{2}+\left|\frac{\partial}{\partial \rho} g_{\mu}\right|^{2} \mathrm{~d} R \mathrm{~d} \rho \\
& \quad-\frac{\sigma^{2}}{2} \int_{\Omega} d^{\prime}\left[g_{\mu}\right] \frac{\partial}{\partial \rho} g_{\mu} \frac{\partial}{\partial \rho} y \mathrm{~d} R \mathrm{~d} \rho .
\end{aligned}
$$

Considering the last integral, we calculate

$$
\begin{aligned}
& -\frac{\sigma^{2}}{2} \int_{\Omega} d^{\prime}\left[g_{\mu}\right] \frac{\partial}{\partial \rho} g_{\mu} \frac{\partial}{\partial \rho} y \mathrm{~d} R \mathrm{~d} \rho=-\frac{\sigma^{2}}{2} \int_{\Omega} d^{\prime}\left[g_{\mu}\right] \frac{\partial}{\partial \rho} g_{\mu} \frac{\partial}{\partial \rho}\left(\frac{\partial}{\partial R} g_{\mu}\right) \mathrm{d} R \mathrm{~d} \rho \\
& =\frac{\sigma^{2}}{2} \int_{\Omega} \frac{\partial}{\partial R} d^{\prime}\left[g_{\mu}\right]\left|\frac{\partial}{\partial \rho} g_{\mu}\right|^{2} \mathrm{~d} R \mathrm{~d} \rho+\frac{\sigma^{2}}{2} \int_{\Omega} d^{\prime}\left[g_{\mu}\right] \frac{\partial}{\partial R}\left(\frac{\partial}{\partial \rho} g_{\mu}\right) \frac{\partial}{\partial \rho} g_{\mu} \mathrm{d} R \mathrm{~d} \rho,
\end{aligned}
$$


and therefore,

$$
-\frac{\sigma^{2}}{2} \int_{\Omega} d^{\prime}\left[g_{\mu}\right] \frac{\partial}{\partial \rho} g_{\mu} \frac{\partial}{\partial \rho} y \mathrm{~d} R \mathrm{~d} \rho=\frac{\sigma^{2}}{4} \int_{\Omega} \frac{\partial}{\partial R} d^{\prime}\left[g_{\mu}\right]\left|\frac{\partial}{\partial \rho} g_{\mu}\right|^{2} \mathrm{~d} R \mathrm{~d} \rho .
$$

This gives us the following estimate for $\|y\|_{L^{2}(\Omega)}$ (with a constant depending on $a\left[g_{\mu}\right]$, $\tilde{c}\left[g_{\mu}\right]$ and their derivatives)

$$
\int_{\Omega} y(t)^{2} \mathrm{~d} R \mathrm{~d} \rho \leq \int_{\Omega} h(0)^{2} \mathrm{~d} R \mathrm{~d} \rho+C \int_{0}^{t} \int_{\Omega} y^{2}+g_{\mu}^{2}+\left|\frac{\partial}{\partial \rho} g_{\mu}\right|^{2} \mathrm{~d} R \mathrm{~d} \rho \mathrm{d} s .
$$

We use similar arguments for $z=\frac{\partial}{\partial \rho} g_{\mu}$. For a suitable $C$, which depends on $a\left[g_{\mu}\right]$, $\tilde{c}\left[g_{\mu}\right], d\left[g_{\mu}\right]$ and their derivatives (but not on $\mu$ ), we obtain an estimate for the $L^{2}$ norm of $z$ :

$$
\int_{\Omega} z(t)^{2} \mathrm{~d} R \mathrm{~d} \rho \leq \int_{\Omega} h(0)^{2} \mathrm{~d} R \mathrm{~d} \rho+C \int_{0}^{t} \int_{\Omega} z^{2}+g_{\mu}^{2}+\left|\frac{\partial}{\partial R} g_{\mu}\right|^{2} \mathrm{~d} R \mathrm{~d} \rho \mathrm{d} s .
$$

We add (27), (31) and (32) to obtain

$$
\begin{aligned}
\int_{\Omega} & g_{\mu}(\rho, R, t)^{2}+y(\rho, R, t)^{2}+z(\rho, R, t)^{2} \mathrm{~d} R \mathrm{~d} \rho \\
& +\frac{\sigma^{2}}{2} \int_{0}^{t} \int_{\Omega} z(\rho, R, s)^{2} \mathrm{~d} R \mathrm{~d} \rho \mathrm{d} s \\
\leq & C \int_{0}^{t} \int_{\Omega} y(\rho, R, s)^{2}+g_{\mu}(\rho, R, s)^{2}+z(\rho, R, s)^{2} \mathrm{~d} R \mathrm{~d} \rho \mathrm{d} s \\
& +\int_{\Omega} g(\rho, R, 0)^{2}+y(\rho, R, 0)^{2}+z(\rho, R, 0)^{2} \mathrm{~d} R \mathrm{~d} \rho,
\end{aligned}
$$

where $C$ does not depend on $\mu$. Using Gronwall's lemma gives the following estimates (independent of $\mu$ )

$$
\left\|g_{\mu}\right\|_{L^{\infty}\left(0, T ; L^{2}(\Omega)\right)} \leq C, \quad\left\|\frac{\partial}{\partial \rho} g_{\mu}\right\|_{L^{\infty}\left(0, T ; L^{2}(\Omega)\right)} \leq C,\left\|\frac{\partial}{\partial R} g_{\mu}\right\|_{L^{\infty}\left(0, T ; L^{2}(\Omega)\right)} \leq C .
$$

Step 4: The limit $\mu \rightarrow 0$. Let $g_{\mu}$ solution of (22a)-(22b) with $L\left[g_{\mu}\right]=a\left[g_{\mu}\right]$ and $K\left[g_{\mu}\right]=\tilde{c}\left[g_{\mu}\right]$. We can estimate $\left\|\frac{\partial}{\partial t} g_{\mu}\right\|_{L^{2}\left(0, T ; H^{-1}(\Omega)\right)}$, using the norm of operators $\left\|\frac{\partial}{\partial t} g_{\mu}\right\|_{H^{-1}(\Omega)}=\sup _{\|v\|_{H^{1}(\Omega)}=1}\left|\left\langle\frac{\partial}{\partial t} g_{\mu}, v\right\rangle\right|$.

For a suitable $C \geq\left(\left\|\frac{\partial}{\partial R} a[g]\right\|_{\infty}\right)^{\frac{1}{2}}+\left(\left\|\frac{\partial}{\partial \rho} \tilde{c}[g]\right\|_{\infty}\right)^{\frac{1}{2}}+\frac{\sigma^{2}}{2}+1$, we obtain

$$
\left|\left\langle\frac{\partial}{\partial t} g_{\mu}, v\right\rangle\right| \leq\left\|a\left[g_{\mu}\right]\right\|_{\infty} \int_{\Omega}\left(g_{\mu}^{2}+\left|\frac{\partial}{\partial R} v\right|^{2}\right) \mathrm{d} R \mathrm{~d} \rho
$$




$$
\begin{aligned}
& +\left\|\tilde{c}\left[g_{\mu}\right]\right\|_{\infty} \int_{\Omega}\left(g_{\mu}^{2}+\left|\frac{\partial}{\partial \rho} v\right|^{2}\right) \mathrm{d} R \mathrm{~d} \rho \\
& +\frac{\sigma^{2}}{2}\left\|d\left[g_{\mu}\right]\right\|_{\infty} \int_{\Omega}\left|\frac{\partial}{\partial \rho} g_{\mu}\right|^{2}+\left|\frac{\partial}{\partial \rho} v\right|^{2} \mathrm{~d} R \mathrm{~d} \rho \\
& +\mu \times \int_{\Omega}\left|\nabla g_{\mu}\right|^{2}+|\nabla v|^{2} \mathrm{~d} R \mathrm{~d} \rho \\
& \leq C\left(\left\|g_{\mu}\right\|_{H^{1}(\Omega)}\right)\|v\|_{H^{1}(\Omega)} .
\end{aligned}
$$

This implies

$$
\left\|\frac{\partial}{\partial t} g_{\mu}\right\|_{L^{2}\left(0, T ; H^{-1}(\Omega)\right)} \leq C \text { and } \int_{0}^{T}\left\|g_{\mu}\right\|_{H^{1}(\Omega)}^{2} \mathrm{~d} t=C\left\|g_{\mu}\right\|_{L^{2}\left(0, T ; H^{1}(\Omega)\right)} \leq C,
$$

where $C$ does not depend on $\mu$. Estimates (34) and (35) allow us to apply Aubin-Lions lemma and conclude the existence of a subsequence of $\left(g_{\mu}\right)$ such that for $\mu \rightarrow 0$,

$$
\begin{gathered}
g_{\mu} \rightarrow g \text { strongly in } L^{2}\left(0, T ; L^{2}(\Omega)\right), \\
g_{\mu} \rightarrow g \text { weakly in } L^{2}\left(0, T ; H^{1}(\Omega)\right), \\
\frac{\partial}{\partial t} g_{\mu} \rightarrow \frac{\partial}{\partial t} g \text { weakly in } L^{2}\left(0, T ; H^{-1}(\Omega)\right) .
\end{gathered}
$$

Furthermore, by direct computation, we obtain

$$
\begin{aligned}
\left\|\tilde{c}[g] g-\tilde{c}\left[g_{\mu}\right] g_{\mu}\right\|_{L^{2}\left(0, T ; L^{2}(\Omega)\right)} \leq\left\|\tilde{c}[g]\left(g-g_{\mu}\right)\right\|_{L^{2}\left(0, T ; L^{2}(\Omega)\right)} \\
+\left\|\left(\tilde{c}[g]-\tilde{c}\left[g_{\mu}\right]\right) g_{\mu}\right\|_{L^{2}\left(0, T ; L^{2}(\Omega)\right)} .
\end{aligned}
$$

The first term on the right side of the previous inequality goes to 0 when $\mu \rightarrow 0$ because $\tilde{c}\left[g_{\mu}\right]$ is bounded and $g_{\mu} \rightarrow g$ strongly in $L^{2}\left(0, T ; L^{2}(\Omega)\right)$. Using CauchySchwartz's inequality and that the domain $\Omega$ is bounded, yields

$$
\begin{aligned}
& \left\|\left(\tilde{c}[g]-\tilde{c}\left[g_{\mu}\right]\right) g_{\mu}\right\|_{L^{1}\left(0, T ; L^{1}(\Omega)\right)}=\int_{0}^{T} \int_{\Omega} \mid \int_{\Omega}\left(\alpha h_{1}\left(\rho_{j}-\rho\right)\right. \\
& \left.\quad+\beta\left\langle h_{2}\left(\rho_{j}-\rho\right)\right\rangle\right) \\
& \quad \times w\left(R-R_{j}\right)\left(g\left(\rho_{j}, R_{j}, t\right)-g_{\mu}\left(\rho_{j}, R_{j}, t\right) \mathrm{d} \rho_{j} \mathrm{~d} R_{j}\right) \mid g_{\mu}(\rho, R, t) \mathrm{d} \rho \mathrm{d} R \mathrm{~d} t \\
& \quad \leq C \int_{0}^{T}\left(\int_{\Omega} g\left(\rho_{j}, R_{j}, t\right)-g_{\mu}\left(\rho_{j}, R_{j}, t\right) \mathrm{d} \rho_{j} \mathrm{~d} R_{j}\right)\left(\int_{\Omega} g_{\mu}(\rho, R, t) \mathrm{d} \rho \mathrm{d} R\right) \mathrm{d} t \\
& \quad \leq C|\Omega|^{\frac{1}{2}}\left\|g_{\mu}-g\right\|_{L^{2}\left(0, T ; L^{2}(\Omega)\right)}^{2} .
\end{aligned}
$$

The constant is bounded from above by the $L^{\infty}$-norm of $h$ and $w$; hence, this term goes to 0 as $\mu \rightarrow 0$. 
Since $c\left[g_{\mu}\right] g_{\mu}$ is bounded, convergence holds in $L^{p}$ for all $p<\infty$. The same argument holds for the difference $\left\|a\left[g_{\mu}\right] g_{\mu}-a[g] g\right\|_{L^{2}\left(0, T ; L^{2}(\Omega)\right)}$. So, we have shown that

$$
\begin{aligned}
& \tilde{c}\left[g_{\mu}\right] g_{\mu} \rightarrow \tilde{c}[g] g \text { strongly in } L^{2}\left(0, T ; L^{2}(\Omega)\right), \\
& a\left[g_{\mu}\right] g_{\mu} \rightarrow a[g] g \text { strongly in } L^{2}\left(0, T ; L^{2}(\Omega)\right) .
\end{aligned}
$$

Therefore, we can pass to the limit $\mu \rightarrow 0$ in Eq. (23) and obtain for all $v \in$ $L^{2}\left(0, T ; H^{1}(\Omega)\right)$

$$
\int_{0}^{T}\left\langle\frac{\partial}{\partial t} g, v\right\rangle \mathrm{d} t=\int_{0}^{T} \int_{\Omega} a[g] g \frac{\partial}{\partial R} v+\tilde{c}[g] g \frac{\partial}{\partial \rho} v-\frac{\sigma^{2}}{2} \frac{\partial}{\partial \rho} g \frac{\partial}{\partial \rho} v \mathrm{~d} R \mathrm{~d} \rho \mathrm{d} t
$$

This completes the proof.

\section{Long-Time Behaviour of Ratings and Strength}

In this section, we study possible steady states of the proposed Elo model and discuss the convergence of the ratings to the strength. We recall that Jabin and Junca (2015) showed that the ratings of players converge to their intrinsic strength in the case $w=1$. This corresponds to the concentration of mass along the diagonal. In our model, the intrinsic strength is continuously increasing in time. Hence, to be able to identify steady states, we consider the shifted Fokker-Planck equation (21a). Throughout this section, we consider the problem in the whole space.

Since the diffusion part in (21a) is singular, the equation is degenerate parabolic. Degenerate Fokker-Planck equations frequently, despite their lack of coercivity, exhibit exponential convergence to equilibrium, a behaviour which has been referred to by Villani as hypocoercivity in Villani (2009). For subsequent research on hypercoercity in linear Fokker-Planck equations, see (Arnold and Erb 2014; Achleitner et al. 2015). Since (21a) is a nonlinear, non-local Fokker-Planck equation these results do not apply here, but it is conceivable that generalisations of this approach can be used in studying the decay to equilibrium for (21a), which is, however, beyond the scope of the present paper. In the following, we present some results on the long-term behaviour of solutions to (21a).

Due to normalisation of the mean value, the only point in which the formation of a steady state is possible is $R_{0}=0$ and $\rho_{0}=0$. Let us assume that we have a measure valued steady state in $(0,0)$, that is $g_{\infty}(\rho, R)=\delta(\rho) \delta(R)$. Then, direct computations using the weak form of (21a) give

$$
\begin{aligned}
0 & =\frac{\partial}{\partial \rho}\left(\phi\left(\rho_{0}, R_{0}\right)\right)\left[\alpha b(0)+\beta\left\langle h_{2}\right\rangle(0)\right]+\frac{\sigma^{2}}{2} w(0) \frac{\partial^{2}}{\partial \rho^{2}}\left(\phi\left(\rho_{0}, R_{0}\right)\right) \\
& =\frac{\sigma^{2}}{2} w(0) \frac{\partial^{2}}{\partial \rho^{2}}\left(\phi\left(\rho_{0}, R_{0}\right)\right) .
\end{aligned}
$$


This equation is not satisfied for all test functions $\phi$. Therefore, we investigate the possibility of having more complex steady states, which have a similar form as the one identified by Junca and Jabin. Let us assume that $g_{\infty}$ is of the form

$$
g_{\infty}(\rho, R)=\delta(\rho) \tilde{g}(R)
$$

or alternatively

$$
g_{\infty}(\rho, R)=\delta(R) \tilde{g}(\rho)
$$

where $\tilde{g}(\cdot)$ in both cases is not a $\delta-$ Dirac.

By direct computation in weak form of (21a) with $\phi(\rho, R)=\rho^{2}$ and $\phi(\rho, R)=R^{2}$, respectively, we compute the following expressions for the second moments of the density function $g(\rho, R, t)$ :

$$
\begin{aligned}
& \frac{\mathrm{d}}{\mathrm{d} t} M_{g, 2, \rho}(t)=\frac{\sigma^{2}}{2} \int_{\mathbb{R}^{4}} w\left(R-R_{j}\right) g(\rho, R, t) g\left(\rho_{j}, R_{j}, t\right) \mathrm{d} R_{j} \mathrm{~d} \rho_{i} \mathrm{~d} R \mathrm{~d} \rho \\
& \quad-\int_{\mathbb{R}^{4}}\left(\rho_{j}-\rho\right)\left[\alpha b\left(\rho_{j}-\rho\right)\right. \\
& \left.\quad+\beta\left\langle h_{2}\left(\rho_{j}-\rho\right)\right\rangle\right] w\left(R-R_{j}\right) g(\rho, R, t) g\left(\rho_{j}, R_{j}, t\right) \mathrm{d} R_{j} \mathrm{~d} \rho_{i} \mathrm{~d} R \mathrm{~d} \rho \\
& \frac{\mathrm{d}}{\mathrm{d} t} M_{g, 2, R}(t)=\int_{\mathbb{R}^{4}} 2 R\left(b\left(\rho-\rho_{j}\right)\right. \\
& \left.\quad-b\left(R-R_{j}\right)\right) w\left(R-R_{j}\right) g(\rho, R, t) g\left(\rho_{j}, R_{j}, t\right) \mathrm{d} R_{j} \mathrm{~d} \rho_{j} \mathrm{~d} R \mathrm{~d} \rho .
\end{aligned}
$$

The analysis of the second moment w.r.t. $\rho$ leads us to conclude that the diffusion prevents the formation of a steady state as in (37) if $w=1$. Indeed, in this case, the first integral in (39) equals $\sigma^{2}$. If at certain time $\bar{t}>0, \rho \simeq \rho_{j}$ or $g(\rho, R, \bar{t})=$ $\delta\left(\rho-\rho_{0}\right) \tilde{g}(R, \bar{t})$, the integral becomes small or vanishes (anyhow smaller than $\sigma^{2}$ ) and then $\frac{\mathrm{d}}{\mathrm{d} t} M_{2, \rho_{i}}(\bar{t}) \geq 0$. Thus, we can conclude that the diffusion prevents the accumulation of the mass in $\rho=0$. For a general choice of $w$, the long-time behaviour of solutions is less clear.

Conversely, the second moment w.r.t. $R$ is decreasing. Due to the symmetry of the functions $b$ and $w$, we can rewrite (40) as

$$
\begin{aligned}
\frac{\mathrm{d}}{\mathrm{d} t} M_{g, 2, R}(t)= & -\int_{\mathbb{R}^{4}}\left(R-R_{j}\right) b\left(R-R_{j}\right) w\left(R-R_{j}\right) g(\rho, R, t) \\
& \times g\left(\rho_{j}, R_{j}, t\right) \mathrm{d} R_{j} \mathrm{~d} \rho_{j} \mathrm{~d} R \mathrm{~d} \rho \leq 0 .
\end{aligned}
$$

This inequality does not contradict the assumption of a steady state of form (38).

In order to evaluate if, with the scaling (20), the rating converges to the intrinsic strength, let us define the energy

$$
E_{2}(t)=\int_{\mathbb{R}^{2}}(\rho-R)^{2} g(\rho, R, t) \mathrm{d} \rho \mathrm{d} R .
$$


We are interested in the evolution of $E_{2}$ and compute

$$
\begin{aligned}
\frac{\mathrm{d}}{\mathrm{d} t} E_{2}(t)= & -2 \int_{\mathbb{R}^{4}}(\rho-R) w\left(R-R_{j}\right) b\left(R-R_{j}\right) g(\rho, R, t) g\left(\rho_{j}, R_{j}, t\right) \mathrm{d} \rho_{j} \mathrm{~d} R_{j} \mathrm{~d} \rho \mathrm{d} R \\
& +2 \int_{\mathbb{R}^{4}}(\rho-R) w\left(R-R_{j}\right) b\left(\rho-\rho_{j}\right) g(\rho, R, t) g\left(\rho_{j}, R_{j}, t\right) \mathrm{d} \rho_{j} \mathrm{~d} R_{j} \mathrm{~d} \rho \mathrm{d} R \\
& +2 \alpha \int_{\mathbb{R}^{4}}(\rho-R) w\left(R-R_{j}\right) b\left(\rho-\rho_{j}\right) g(\rho, R, t) g\left(\rho_{j}, R_{j}, t\right) \mathrm{d} \rho_{j} \mathrm{~d} R_{j} \mathrm{~d} \rho \mathrm{d} R \\
& +2 \beta \int_{\mathbb{R}^{4}}(\rho-R) w\left(R-R_{j}\right)\left\langle h_{2}\left(\rho-\rho_{j}\right)\right\rangle g(\rho, R, t) g\left(\rho_{j}, R_{j}, t\right) \mathrm{d} \rho_{j} \mathrm{~d} R_{j} \mathrm{~d} \rho \mathrm{d} R \\
& +\sigma^{2} \int_{\mathbb{R}^{2}} d[g] g(\rho, R, t) \mathrm{d} \rho \mathrm{d} R .
\end{aligned}
$$

For general functions $w$, it is not possible to determine the signs of the respective integrals. Therefore, we consider the case $w=1$ only. For all odd functions $b(\cdot)$ (the same holds true for $\left.\left\langle h_{2}\left(\rho-\rho_{j}\right)\right\rangle\right)$, we are able to show that

$$
\begin{aligned}
& \int_{\mathbb{R}^{4}} \rho b\left(\rho_{j}-\rho\right) g(\rho, R, t) g\left(\rho_{j}, R_{j}, t\right) \mathrm{d} \rho_{j} \mathrm{~d} R_{j} \mathrm{~d} \rho \mathrm{d} R \\
& =\frac{1}{2} \int_{\mathbb{R}^{4}} \rho\left(b\left(\rho_{j}-\rho\right)-b\left(\rho-\rho_{j}\right)\right) g(\rho, R, t) g\left(\rho_{j}, R_{j}, t\right) \mathrm{d} \rho_{j} \mathrm{~d} R_{j} \mathrm{~d} \rho \mathrm{d} R \\
& =-\frac{1}{2} \int_{\mathbb{R}^{4}}\left(\rho_{j}-\rho\right) b\left(\rho_{j}-\rho\right) g(\rho, R, t) g\left(\rho_{j}, R_{j}, t\right) \mathrm{d} \rho_{j} \mathrm{~d} R_{j} \mathrm{~d} \rho \mathrm{d} R \\
& \quad \leq 0
\end{aligned}
$$

and $\int_{\Omega^{2}} \rho b\left(R-R_{j}\right) g(\rho, R, t) g\left(\rho_{j}, R_{j}, t\right) \mathrm{d} \rho_{j} \mathrm{~d} R_{j} \mathrm{~d} \rho \mathrm{d} R=0$. In this case we can rewrite Eq. (42) as

$$
\begin{aligned}
\frac{\mathrm{d}}{\mathrm{d} t} E_{2}(t)=- & \int_{\mathbb{R}^{4}}\left(R-R_{j}\right) b\left(R-R_{j}\right) g(\rho, R, t) g\left(\rho_{j}, R_{j}, t\right) \mathrm{d} \rho_{j} \mathrm{~d} R_{j} \mathrm{~d} \rho \mathrm{d} R \\
& -\int_{\mathbb{R}^{4}}\left(\rho-\rho_{j}\right) b\left(\rho-\rho_{j}\right) g(\rho, R, t) g\left(\rho_{j}, R_{j}, t\right) \mathrm{d} \rho_{j} \mathrm{~d} R_{j} \mathrm{~d} \rho \mathrm{d} R \\
& -\alpha \int_{\mathbb{R}^{4}}\left(\rho-\rho_{j}\right) b\left(\rho-\rho_{j}\right) g(\rho, R, t) g\left(\rho_{j}, R_{j}, t\right) \mathrm{d} \rho_{j} \mathrm{~d} R_{j} \mathrm{~d} \rho \mathrm{d} R \\
& -2 \beta \int_{\mathbb{R}^{4}}\left(\rho-\rho_{j}\right)\left\langle h_{2}\left(\rho-\rho_{j}\right)\right\rangle g(\rho, R, t) g\left(\rho_{j}, R_{j}, t\right) \mathrm{d} \rho_{j} \mathrm{~d} R_{j} \mathrm{~d} \rho \mathrm{d} R \\
& +\sigma^{2} .
\end{aligned}
$$

Again we would like to know if a concentration of mass along the diagonal is possible. Let us assume that at certain time the solution is $g(\rho, R, t)=\delta(\rho-R) \tilde{g})(\rho, R, t)$. If 
we insert this claim in (43), we obtain

$$
\frac{\mathrm{d}}{\mathrm{d} t} E_{2}(t)=\sigma^{2}>0
$$

It shows that the diffusion counteracts the accumulation of the mass along the diagonal. On the other hand, the four integrals in (43) are strictly negative. Hence, if $\sigma^{2}$ is small enough, the distance between rating and intrinsic strength becomes small, and the diffusive term can be controlled. This indicates concentration of the mass in a certain neighbourhood of the diagonal in the long run.

\section{Numerical Simulations}

In this section, we discuss the numerical discretisation of the Boltzmann equation (11) and the shifted Fokker-Planck equation (21a). We initialise the distribution of players with respect to their strength and rating with values from the unit interval and consider appropriately shifted interaction rules to ensure that the distribution remains inside the unit square for all times $t>0$.

\subsection{Monte Carlo Simulations of the Boltzmann Equation}

We use the classical Monte Carlo method to compute a series of realisations of the Boltzmann equation (11). In the direct Monte Carlo method, also known as Bird's scheme, pairs of players are randomly and non-exclusively selected for two-player games. The outcome of the game is determined by (6). Note that we consider the following shifted interaction rules for the ratings, to ensure that $\rho \in[0,1]$ and $R \in$ $[0,1]$ :

$$
\begin{aligned}
& \rho_{i}^{*}=\rho_{i}+\gamma \tilde{h}\left(\rho_{j}-\rho_{i}\right) w\left(R_{i}-R_{j}\right)+\eta \\
& \rho_{j}^{*}=\rho_{j}+\gamma \tilde{h}\left(\rho_{i}-\rho_{j}\right) w\left(R_{i}-R_{j}\right)+\tilde{\eta},
\end{aligned}
$$

where $\tilde{h}=b\left(\rho_{j}-\rho_{i}\right)$. The microscopic interactions are simulated as follows: the outcome of the game $S_{i j}$ is the realisation of a discrete distribution function, which takes the value $\{-1,1\}$ with probability $\left\{b\left(\rho_{i}-\rho_{j}\right), 1-b\left(\rho_{i}-\rho_{j}\right)\right\}$. The random variables $\eta$ are generated such that they assume values $\eta= \pm 0.025$ with equal probability, and the parameter $\gamma$ is set to 0.05 . Further information on Monte Carlo methods for Boltzmann-type equations can be found in Pareschi and Toscani (2013).

In each simulation, we consider $N=5000$ players and compute the steady-state distribution by performing $10^{8}$ time steps. The result is then averaged over another $10^{5}$ time steps. We perform $M=10$ realisations and compute the density from the averaged steady states. 


\subsection{Finite Volume Discretisation and Simulations of the Nonlinear Fokker-Planck Equation}

The solver for the Fokker-Planck equation is based on a Strang splitting and an upwind finite volume scheme. We recall that we discretise the shifted Fokker-Planck equation (21a), which allows us to perform simulations on a bounded domain. Because of the splitting, we consider the interactions in the rating and the strength variable separately. We define two operators, which correspond to

$\left(\mathcal{S}_{1}\right)$ Interaction step in the strength variable $R$ :

$$
\frac{\partial g^{*}}{\partial t}(\rho, R, t)=-\frac{\partial}{\partial \rho}\left(c[\tilde{g}] g^{*}(\rho, R, t)\right)+\frac{\sigma^{2}}{2} d[\tilde{g}] \frac{\partial^{2}}{\partial \rho^{2}}\left(g^{*}(\rho, R, t)\right)
$$

subject to the initial condition $g^{*}(\rho, R, t)=\tilde{g}(\rho, R, t)$. Note that we compute the interaction integrals using $\tilde{g}$, which corresponds to the solution at the previous time step in the full splitting scheme.

$\left(\mathcal{S}_{2}\right)$ Interaction step in the rating variable $\rho$ :

$$
\frac{\partial g^{\diamond}}{\partial t}(\rho, R, t)=-\frac{\partial}{\partial R}\left(a\left[g^{*}\right] g^{\diamond}(\rho, R, t)\right)
$$

We approximate all integrals, which appear in the interaction coefficients using the trapezoidal rule.

Let $\hat{g}^{k}$ denote the solution at time $t^{k}=k \Delta t$, where $\Delta t$ corresponds to the time step size. Then, the Strang splitting results in the scheme

$$
\hat{g}^{k+1}(\rho, R)=\mathcal{S}_{2}\left(\hat{g}^{*, k+1}, \frac{\Delta t}{2}\right) \circ \mathcal{S}_{1}\left(\hat{g}^{\diamond, k+\frac{1}{2}}, \Delta t\right) \circ \mathcal{S}_{2}\left(\hat{g}^{k}, \frac{\Delta t}{2}\right),
$$

where the superscripts denote the solutions of $g^{*}$ and $g^{\diamond}$ at the discrete time steps $t^{k+1}=(k+1) \Delta t$ and $t^{k+\frac{1}{2}}=\left(k+\frac{1}{2}\right) \Delta t$. We use a conservative upwind finite volume discretisation to discretise the respective operators. The corresponding explicit-in-time upwind finite volume methods are given by

$$
\hat{g}_{j}^{n+1}=\hat{g}_{j}^{n}+\lambda_{1}\left(\hat{c}_{j+\frac{1}{2}}-\hat{c}_{j-\frac{1}{2}}\right)+\lambda_{2}\left(\hat{d}_{j+\frac{1}{2}}-\hat{d}_{j-\frac{1}{2}}\right),
$$

where $\hat{c}$ is the upwind flux and the diffusive flux is given $\hat{d}_{j+\frac{1}{2}}=D\left(\hat{g}_{j+1}\right) \hat{g}_{j+1}-$ $D\left(\hat{g}_{j}\right) \hat{g}_{j}$. Here, $\lambda_{1}=\Delta t / \Delta x$ and $\lambda_{2}=\Delta t / \Delta x^{2}$.

\subsection{Computational Experiments}

All micro- and macroscopic simulations are performed on the domain $[0,1] \times[0,1]$ with no-flux boundary conditions. In the case of a general interaction function, the interaction rate function $w\left(r_{i}-r_{j}\right)$ is a piecewise constant function given by 


$$
w(z)= \begin{cases}1 & \text { if }|z| \leq 0.1 \\ 0 & \text { otherwise }\end{cases}
$$

\subsubsection{All-Play-All Tournaments}

We start by investigating the long-time behaviour of the Elo model with $w=1$, $\alpha=0.1$ and $\beta=0$ in (7). Hence, players have the same probability to play against another independent of their respective ratings. We have seen in Sect. 4 that we expect a measure valued solution in the case of no diffusion. However, we cannot show convergence of solutions to a measure valued steady state if stochastic fluctuations influence the intrinsic strength. In the following, we compare computed steady states of the Boltzmann as well as the Fokker-Planck equation in the case of diffusion and no diffusion. We start with a uniform distribution of agents in the micro- and the macroscopic situation. Figure 2 and Fig. 3 confirm the expected formation of a Delta Dirac at the centre of mass in the case of no diffusion. If the individual strength is also influenced by stochastic fluctuations, the steady state is smoothed out with respect to the rating as well. The resulting steady states are Gaussian like profiles in the microand macroscopic simulations (see Figs. 2 and 3). Figure 3 also shows the decay of the energy $E_{2}$ in time.

\subsubsection{Competitions of Players with Similar Ratings}

Assigning initial ratings to players in the Elo rating is a delicate issue, since inaccurate initial ratings may influence the ability of the rating to converge to a 'good' rating of players reflecting their intrinsic strengths. We show the difficulties in this case by studying the dynamics if players with close ratings compete.

We set the interaction rate function to (45) — hence, individuals only play against each other, if the difference between their ratings is small. We consider two groups of players with different strength and rating levels as initial distribution. The first group is underrated, that is all players have rating $R=0.2$, but their strength is distributed as $\rho \in \mathcal{N}(0.75,0.1)$. The second group is overrated, with rating $R=0.9$ and a uniform distribution in strength. We use this initial configuration in two computational experiments.

In the first, we choose the learning parameters $\alpha=0.1$ and $\beta=0$. We see that the two groups remain separated due to their different ratings in this case (see Fig. 4). However, players compete within their own group and since $\beta=0$ the overall rating improves. In the overrated group the strongest players accumulate at the highest possible rating, while the underrated group forms a diagonal pattern. Here, the underrated players evolve to the maximum possible rating level.

In the second experiment, using the same initial configuration, but $\alpha=0.1$ and $\beta=0.05$ the steady-state profile looks totally different. In this setting, stronger players loose strength, when loosing against a weaker opponent. Therefore, the ratings of the overrated group decrease, while the ratings of the underrated group increase. After a while the two groups merge, accumulating on a diagonal which underestimates the intrinsic strength of players by approximately 0.1 (see Fig. 5). 


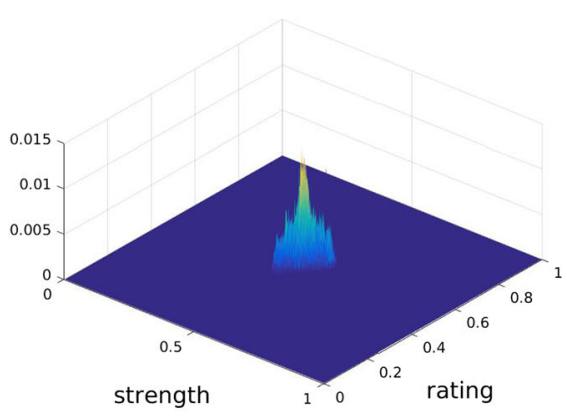

(a)

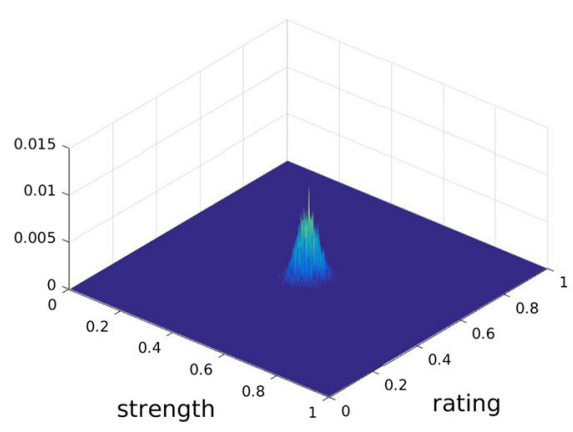

(c)

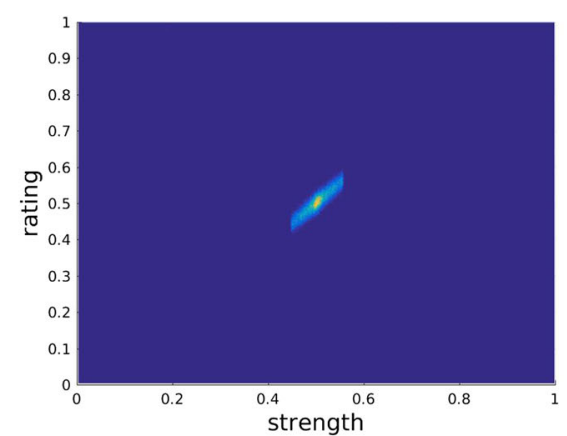

(b)

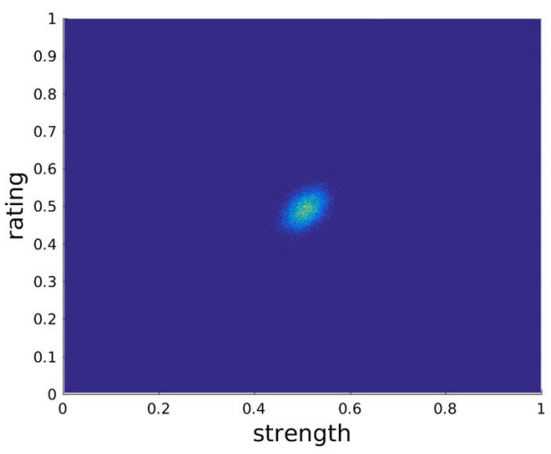

(d)

Fig. 2 Computational steady state of the Boltzmann model for $w=1$ in the case of no diffusion, $\eta=\bar{\eta}=0$, and small diffusion in the strength $\eta=\bar{\eta}=0.025$. a Steady state-no diffusion. b Steady state (top view) no diffusion. $\mathbf{c}$ Steady state-diffusion $v=0.025$. d Steady state (top view) — diffusion $v=0.025$

These examples show the importance of the initial ratings as well as the influence of the adapted learning mechanism.

\subsubsection{Foul Play}

Finally, we consider a series of games, in which one player, without loss of generality the first one, is playing unfairly, e.g. through cheating, doping or bribing of referees. This means that the outcome of every microscopic game which involves this player is biased in their favour. In particular, we assume that the probability of winning is increased by a factor $\tilde{b}$ for player 1 and decreased by $\tilde{b}$ for the other contestant. Figure 6 shows the stationary profile in the case of a uniform initial distribution of agents, $\alpha=0.1, \beta=0, w=1$ and $\tilde{b}=0.2$. The star indicates the position of the unfair first player. While the distribution of players with respect to their ratings and their strengths accumulates along the diagonal, we see that the first player is rated higher than implied by his or her strength. 


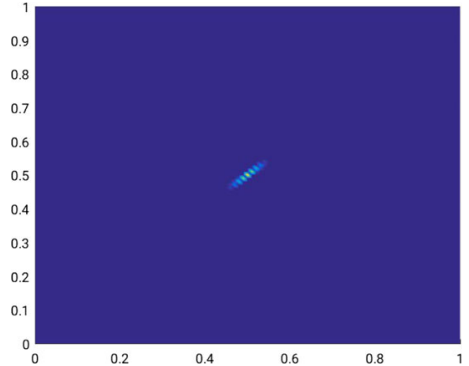

(a)

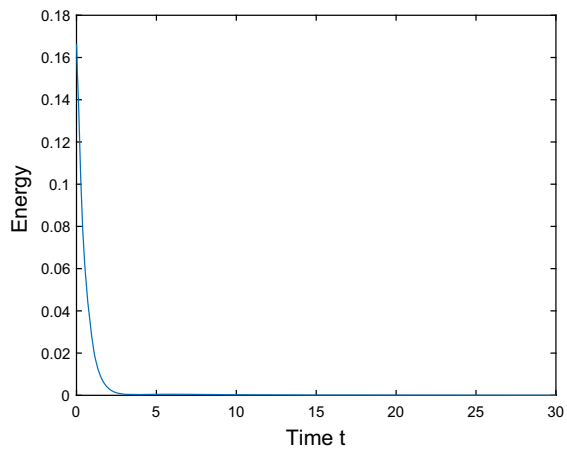

(c)

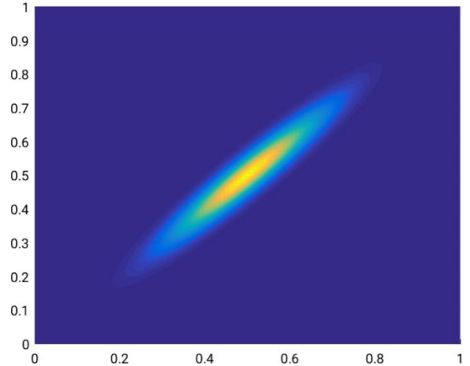

(b)

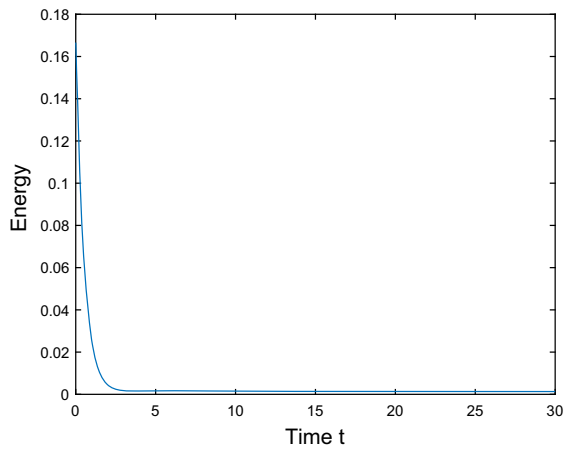

(d)

Fig. 3 Computational steady state of the Fokker-Planck model and energy decay for $w=1$ in the case of no and little diffusion strength. a Steady state (top view) — no diffusion. b Steady state (top view) —with diffusion. $\mathbf{c}$ Energy decay in the case of no diffusion. d Energy decay in the case of diffusion
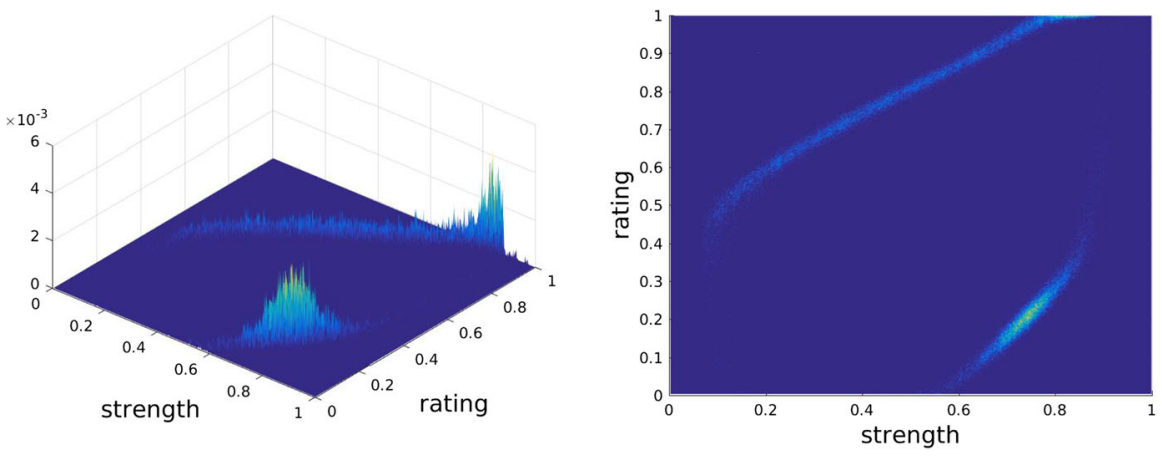

Fig. 4 Computed stationary profiles in competitions of players with similar ratings in case of two initially separated groups (one underrated with high strength but low rating and one overrated with variable strength but rating 0.9). Due to the limited interaction between the groups and the chosen learning mechanism, they remain separated 

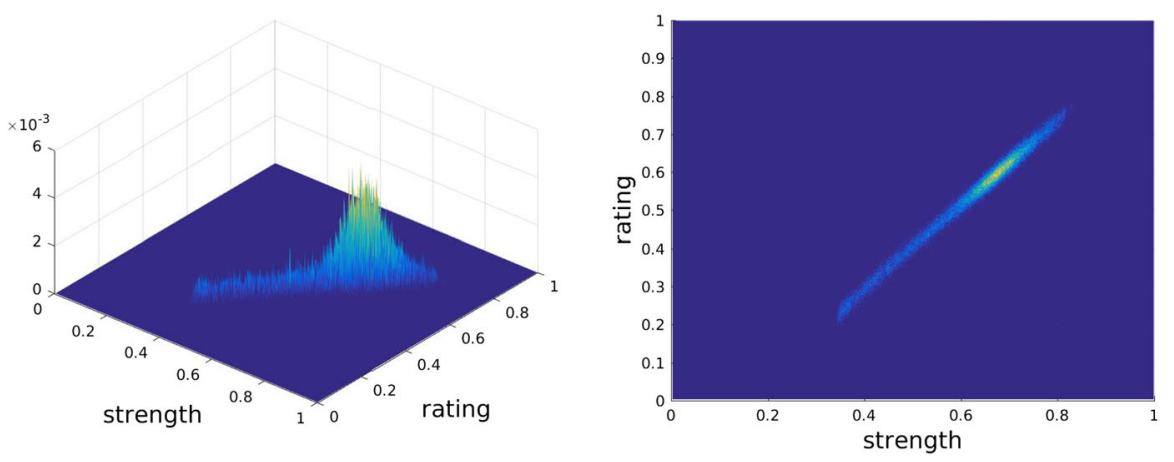

Fig. 5 Computed stationary profiles in competitions of players with similar ratings in case of two initially separated groups (one underrated with high strength but low rating and one overrated with variable strength but rating 0.9). Despite the limited interaction between the groups, the adapted learning mechanism leads to convergence of the ratings to a slightly shifted diagonal

Fig. 6 Computed stationary profile in a foul play where the first player has an unfair advantage in each game. We observe that the ratings and strength all players except the first one converge. The cheating player (indicated by a star) ends up with a higher rating than it is supposed to have

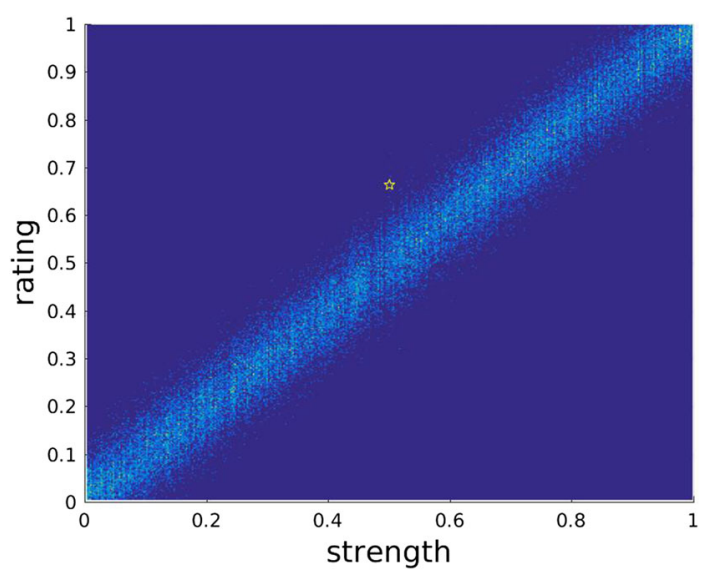

Acknowledgements The authors thank Martin Burger for the useful discussion during the Warwick EPSRC symposium workshop on 'Emerging PDE models in socio-economic sciences'. The authors are grateful to two anonymous referees for constructive comments and remarks.

Funding BD has been supported by the Leverhulme Trust research project grant 'Novel discretisations for higher-order nonlinear PDE' (RPG-2015-69). Part of this research was carried out during a three-month visit of the second author to the University of Sussex, enabled through financial support by the University of Pavia. MTW acknowledges partial support from the Austrian Academy of Sciences via the New Frontier's Grant NST 0001 and the EPSRC by the Grant EP/P01240X/1.

\section{Compliance with Ethical Standards}

Conflict of interest The authors declare that they have no conflict of interest.

Open Access This article is distributed under the terms of the Creative Commons Attribution 4.0 International License (http://creativecommons.org/licenses/by/4.0/), which permits unrestricted use, distribution, and reproduction in any medium, provided you give appropriate credit to the original author(s) and the source, provide a link to the Creative Commons license, and indicate if changes were made. 


\section{Appendix A: Derivation of the Fokker-Planck Equation}

In this section, we derive the limiting Fokker-Planck equation in the case $\gamma \rightarrow 0$, $\sigma_{\eta} \rightarrow 0$ such that $\sigma_{\eta}^{2} / \gamma=: \sigma^{2}$ is kept fixed. Based on the interaction rules (6), which define the outcome of a game, we compute the expected values of the following quantities:

$$
\begin{aligned}
\left\langle\left(R_{i}^{*}-R_{i}\right)\right\rangle & =\gamma\left(b\left(\rho_{i}-\rho_{j}\right)-b\left(R_{i}-R_{j}\right)\right) \\
\left\langle\left(\left(R_{i}^{*}-R_{i}\right)^{2}\right)\right\rangle & =\gamma^{2}\left(b\left(\rho_{i}-\rho_{j}\right)-b\left(R_{i}-R_{j}\right)\right)^{2} ; \\
\left\langle\left(\rho_{i}^{*}-\rho_{i}\right)\right\rangle= & \gamma\left(\alpha h_{1}\left(\rho_{j}-\rho_{i}\right)+\beta\left\langle h_{2}\left(\rho_{j}-\rho_{i}\right)\right\rangle\right) \\
\left\langle\left(\rho_{i}^{*}-\rho_{i}\right)^{2}\right\rangle= & \gamma^{2}\left(\alpha h_{1}\left(\rho_{j}-\rho_{i}\right)+\beta\left\langle h_{2}\left(\rho_{j}-\rho_{i}\right)\right\rangle\right)^{2}+\sigma_{\eta}^{2} \\
\left\langle\left(\rho_{i}^{*}-\rho_{i}\right)\left(R_{i}^{*}-R_{i}\right)\right\rangle= & \gamma^{2}\left(\alpha h_{1}\left(\rho_{j}-\rho_{i}\right)+\beta\left\langle h_{2}\left(\rho_{j}-\rho_{i}\right)\right\rangle\right) \\
& \times\left(b\left(\rho_{i}-\rho_{j}\right)-b\left(R_{i}-R_{j}\right)\right) .
\end{aligned}
$$

Using Taylor expansion of $\phi\left(\rho_{i}^{*}, R_{i}^{*}\right)$ up to order two around $\left(\rho_{i}, R_{i}\right)$, we obtain

$$
\begin{aligned}
\left\langle\phi\left(\rho_{i}^{*}, R_{i}^{*}\right)-\phi\left(\rho_{i}, R_{i}\right)\right\rangle \\
=\left\langle R_{i}^{*}-R_{i}\right\rangle \frac{\partial}{\partial R_{i}} \phi\left(\rho_{i}, R_{i}\right)+\left\langle\rho_{i}^{*}-\rho_{i}\right\rangle \frac{\partial}{\partial \rho_{i}} \phi\left(\rho_{i}, R_{j}\right) \\
\quad+\frac{1}{2}\left[\left\langle\left(R_{i}^{*}-R_{i}\right)^{2}\right\rangle \frac{\partial^{2}}{\partial R_{i}^{2}} \phi\left(\rho_{i}, R_{i}\right)+\left\langle\left(\rho_{i}^{*}-\rho_{i}\right)^{2}\right\rangle \frac{\partial^{2}}{\partial \rho_{i}^{2}} \phi\left(\rho_{i}, R_{i}\right)\right. \\
\left.\quad+2\left\langle\left(\rho_{i}^{*}-\rho_{i}\right)\left(R_{i}^{*}-R_{i}\right)\right\rangle \frac{\partial^{2}}{\partial \rho_{i} \partial R_{i}} \phi\left(\rho_{i}, R_{i}\right)\right] \\
\quad+\mathcal{R}_{\gamma}\left(\phi, \rho_{i}^{*}, R_{i}^{*}, \rho_{i}, R_{i}, \tau\right),
\end{aligned}
$$

where the remainder term $\mathcal{R}_{\gamma}$ is given by

$$
\begin{aligned}
& \mathcal{R}_{\gamma} \\
& \quad=\left(\begin{array}{c}
\rho_{i}^{*}-\rho_{i} \\
R_{i}^{*}-R_{i}
\end{array}\right)^{T}\left(\begin{array}{cc}
\frac{\partial^{2}}{\partial \rho_{i}^{2}} \phi\left(\overline{\rho_{i}}, \overline{R_{i}}\right)-\frac{\partial^{2}}{\partial \rho_{i}^{2}} \phi\left(\rho_{i}, R_{i}\right) & \frac{\partial^{2}}{\partial \rho_{i} \partial R_{i}} \phi\left(\overline{\rho_{i}}, \overline{R_{i}}\right)-\frac{\partial^{2}}{\partial \rho_{i} \partial R_{i}} \phi\left(\rho_{i}, R_{i}\right) \\
\frac{\partial^{2}}{\partial \rho_{i} \partial R_{i}} \phi\left(\overline{\rho_{i}}, \overline{R_{i}}\right)-\frac{\partial^{2}}{\partial \rho_{i} \partial R_{i}} \phi\left(\rho_{i}, R_{i}\right) & \frac{\partial^{2}}{\partial R_{i}^{2}} \phi\left(\overline{\rho_{i}}, \overline{R_{i}}\right)-\frac{\partial^{2}}{\partial R_{i}^{2}} \phi\left(\rho_{i}, R_{i}\right)
\end{array}\right)\left(\begin{array}{c}
\rho_{i}^{*}-\rho_{i} \\
R_{i}^{*}-R_{i}
\end{array}\right),
\end{aligned}
$$

for some $0 \leq \theta_{1}, \theta_{2} \leq 1$ with $\overline{\rho_{i}}$ and $\overline{R_{i}}$ defined as

$$
\overline{\rho_{i}}=\theta_{1} \rho_{i}+\left(1-\theta_{1}\right) \rho_{i}^{*} \text { and } \overline{R_{i}}=\theta_{2} R_{i}+\left(1-\theta_{2}\right) R_{i}^{*}
$$


Next we rescale time as $\tau=\gamma t$ and insert the expansion in (11). This yields

$$
\begin{aligned}
& \frac{\mathrm{d}}{d \tau} \int_{\mathbb{R}^{2}} \phi\left(\rho_{i}, R_{j}\right) f_{\gamma}\left(\rho_{i}, R_{i}, \tau\right) \mathrm{d} R_{i} \mathrm{~d} \rho_{i} \\
& =\frac{1}{2 \gamma} \int_{\mathbb{R}^{2}} \tilde{\mathcal{R}}_{\gamma}\left(\phi, \rho_{i}^{*}, R_{i}^{*}, \rho_{i}, R_{i}, \tau\right) f_{\gamma}\left(\rho_{i}, R_{i}, \tau\right) \mathrm{d} R_{i} \mathrm{~d} \rho_{i} \\
& \quad+\int_{\mathbb{R}^{4}}\left[\frac{\partial}{\partial R_{i}} \phi\left(\rho_{i}, R_{j}\right)\left(b\left(\rho_{i}-\rho_{j}\right)-b\left(R_{i}-R_{j}\right)\right)\right. \\
& \quad+\frac{\partial}{\partial \rho_{i}} \phi\left(\rho_{i}, R_{j}\right)\left(\alpha h_{1}\left(\rho_{j}-\rho_{i}\right)+\beta\left\langle h_{2}\left(\rho_{j}-\rho_{i}\right)\right\rangle\right) \\
& \left.\quad+\frac{\sigma_{\eta}^{2}}{2 \gamma} \frac{\partial^{2}}{\partial \rho_{i}^{2}} \phi\left(\rho_{i}, R_{j}\right)\right] w\left(R_{i}-R_{j}\right) f_{\gamma}\left(\rho_{i}, R_{i}, \tau\right) f_{\gamma}\left(\rho_{j}, R_{j}, \tau\right) \mathrm{d} R_{j} \mathrm{~d} \rho_{j} \mathrm{~d} R_{i} \mathrm{~d} \rho_{i},
\end{aligned}
$$

where

$$
\begin{aligned}
& \tilde{\mathcal{R}}_{\gamma}\left(\phi, \rho_{i}^{*}, R_{i}^{*}, \rho_{i}, R_{i}, \tau\right)=\gamma^{2} \int_{\mathbb{R}^{2}} \frac{\partial^{2}}{\partial R_{i}^{2}} \phi\left(\rho_{i}, R_{i}\right)\left(b\left(\rho_{i}-\rho_{j}\right)\right. \\
& \left.\quad-b\left(R_{i}-R_{j}\right)\right)^{2} w\left(R_{i}-R_{j}\right) f_{\gamma}\left(\rho_{j}, R_{j}, \tau\right) \mathrm{d} R_{j} \mathrm{~d} \rho_{j} \\
& \quad+\gamma^{2} \int_{\mathbb{R}^{2}} \frac{\partial^{2}}{\partial \rho_{i}^{2}} \phi\left(\rho_{i}, R_{i}\right)\left(\alpha h_{1}\left(\rho_{j}-\rho_{i}\right)\right. \\
& \left.\quad+\beta\left\langle h_{2}\left(\rho_{j}-\rho_{i}\right)\right\rangle\right)^{2} w\left(R_{i}-R_{j}\right) f_{\gamma}\left(\rho_{j}, R_{j}, \tau\right) \mathrm{d} R_{j} \mathrm{~d} \rho_{j} \\
& \quad+2 \gamma^{2} \int_{\mathbb{R}^{2}} \frac{\partial}{\partial \rho_{i} \partial R_{i}} \phi\left(\rho_{i}, R_{i}\right)\left(b\left(\rho_{i}-\rho_{j}\right)-b\left(R_{i}-R_{j}\right)\right)\left(\alpha h_{1}\left(\rho_{j}-\rho_{i}\right)\right. \\
& \left.\quad+\beta\left\langle h_{2}\left(\rho_{j}-\rho_{i}\right)\right\rangle\right) w\left(R_{i}-R_{j}\right) f_{\gamma}\left(\rho_{j}, R_{j}, \tau\right) \mathrm{d} R_{j} \mathrm{~d} \rho_{j} \\
& \quad+\int_{\mathbb{R}^{2}} R_{\gamma} w\left(R_{i}-R_{j}\right) f_{\gamma}\left(\rho_{j}, R_{j}, \tau\right) \mathrm{d} R_{j} \mathrm{~d} \rho_{j} .
\end{aligned}
$$

Next we show that the remainder $\frac{1}{2 \gamma} \int_{\mathbb{R}^{2}} \tilde{\mathcal{R}}_{\gamma}\left(\phi, \rho_{i}^{*}, R_{i}^{*}, \rho_{i}, R_{i}, \tau\right) f_{\gamma}\left(\rho_{i}, R_{i}, \tau\right) \mathrm{d} R_{i} \mathrm{~d} \rho_{i}$ vanishes for $\gamma \rightarrow 0$. Let us assume that $\phi\left(\rho_{i}, R_{i}\right)$ belongs to the space $\mathcal{C}_{2+\delta}\left(\mathbb{R}^{2}\right)=$ $\left\{h: \mathbb{R}^{2} \rightarrow \mathbb{R},\left\|D^{\zeta} h\right\|_{\delta}<+\infty\right\}$, where $0<\delta \leq 1, \zeta$ is a multi-index with $|\zeta| \leq 2$ and the seminorm $\|\cdot\|_{\delta}$ is the usual Hölder seminorm

$$
\|f\|_{\delta}=\sup _{x, y \in \mathbb{R}^{2}} \frac{|f(x)-f(y)|}{|x-y|^{\delta}}
$$

With this choice of $\phi\left(\rho_{i}, R_{i}\right)$, all the terms which contain $\frac{\partial^{2}}{\partial \rho_{i}^{2}} \phi$ and $\frac{\partial^{2}}{\partial R_{i}^{2}} \phi$ vanish using the same arguments as in Toscani (2006), Cordier et al. (2009). Hence, we focus on the mixed derivative $\frac{\partial^{2}}{\partial \rho_{i} \partial R_{i}} \phi\left(\rho_{i}, R_{i}\right)$. Since $\phi\left(\rho_{i}, R_{i}\right) \in \mathcal{C}_{2+\delta}\left(\mathbb{R}^{2}\right)$ and $\|\left(\overline{\rho_{i}}, \overline{R_{i}}\right)-$ 
$\left(\rho_{i}, R_{i}\right)\|\leq\|\left(\rho_{i}^{*}, R_{i}^{*}\right)-\left(\rho_{i}, R_{i}\right) \|$, we have

$$
\left|\frac{\partial^{2}}{\partial \rho_{i} \partial R_{i}} \phi\left(\overline{\rho_{i}}, \overline{R_{i}}\right)-\frac{\partial^{2}}{\partial \rho_{i} \partial R_{i}} \phi\left(\rho_{i}, R_{i}\right)\right| \leq\|\phi\|_{2+\delta}\left\|\left(\rho_{i}^{*}, R_{i}^{*}\right)-\left(\rho_{i}, R_{i}\right)\right\|^{\delta} .
$$

Furthermore, due to (2), (8) and (9),

$$
\begin{aligned}
& \left\|\left(\rho_{i}^{*}, R_{i}^{*}\right)-\left(\rho_{i}, R_{i}\right)\right\| \\
& =\left[\gamma^{2}\left(\alpha h_{1}\left(\rho_{j}-\rho_{i}\right)+\beta\left\langle h_{2}\left(\rho_{j}-\rho_{i}\right)\right\rangle\right)^{2}+\gamma^{2}\left(b\left(\rho_{i}-\rho_{j}\right)-b\left(R_{i}-R_{j}\right)\right)^{2}\right]^{\frac{1}{2}} \\
& \quad \leq C \gamma .
\end{aligned}
$$

Using the previous inequalities, we estimate the mixed term as

$$
\begin{aligned}
& \frac{1}{2 \gamma}\left|\int_{\mathbb{R}^{4}}\left(\frac{\partial^{2} \phi\left(\overline{\rho_{i}}, \overline{R_{i}}\right)}{\partial \rho_{i} \partial R_{i}}-\frac{\partial^{2} \phi\left(\rho_{i}, R_{i}\right)}{\partial \rho_{i} \partial R_{i}}\right)\left\|\left(\frac{\overline{\rho_{i}}}{R_{i}}\right)-\left(\begin{array}{c}
\rho_{i} \\
R_{i}
\end{array}\right)\right\|^{2} w\left(R_{i}-R_{j}\right) f_{\gamma}\left(\rho_{j}, R_{j}, \tau\right) f_{\gamma}\left(\rho_{i}, R_{i}, \tau\right) \mathrm{d} R_{i} \mathrm{~d} \rho_{i} \mathrm{~d} R_{j} \mathrm{~d} \rho_{j}\right| \\
& \quad \leq \frac{1}{2 \gamma} \int_{\mathbb{R}^{4}}\|\phi\|_{2+\delta} \|\left(\rho_{i}^{*}, R_{i}^{*}\right) \\
& \quad-\left(\rho_{i}, R_{i}\right)\left\|^{\delta}\right\|\left(\rho_{i}^{*}, R_{i}^{*}\right)-\left(\rho_{i}, R_{i}\right) \|^{2} f_{\gamma}\left(\rho_{j}, R_{j}, \tau\right) f_{\gamma}\left(\rho_{i}, R_{i}, \tau\right) \mathrm{d} R_{i} \mathrm{~d} \rho_{i} \mathrm{~d} R_{j} \mathrm{~d} \rho_{j} \\
& \quad \leq \frac{1}{2 \gamma} \int_{\mathbb{R}^{4}} C^{\delta}\|\phi\|_{2+\delta} \gamma^{2+\delta} f_{\gamma}\left(\rho_{j}, R_{j}, \tau\right) f_{\gamma}\left(\rho_{i}, R_{i}, \tau\right) \mathrm{d} R_{i} \mathrm{~d} \rho_{i} \mathrm{~d} R_{j} \mathrm{~d} \rho_{j} \\
& \quad \leq \frac{C^{\delta}}{2}\|\phi\|_{2+\delta} \gamma^{1+\delta} .
\end{aligned}
$$

Hence, the remainder term converges to 0 as $\gamma \rightarrow 0$. Therefore, the density $f_{\gamma}\left(\rho_{i}, R_{i}, \tau\right)$ converges to $f\left(\rho_{i}, R_{i}, \tau\right)$ which solves

$$
\begin{aligned}
& \frac{\mathrm{d}}{d \tau} \int_{\mathbb{R}^{2}} \phi\left(\rho_{i}, R_{j}\right) f\left(\rho_{i}, R_{i}, \tau\right) \mathrm{d} R_{i} \mathrm{~d} \rho_{i}= \\
& \quad \int_{\mathbb{R}^{2}} f\left(\rho_{i}, R_{i}, \tau\right)\left\{\frac { \partial } { \partial R _ { i } } \phi ( \rho _ { i } , R _ { j } ) \left[\int _ { \mathbb { R } ^ { 2 } } w ( R _ { i } - R _ { j } ) \left(b\left(\rho_{i}-\rho_{j}\right)\right.\right.\right. \\
& \left.\left.\quad-b\left(R_{i}-R_{j}\right)\right) f\left(\rho_{j}, R_{j}, \tau\right) \mathrm{d} \rho_{j} \mathrm{~d} R_{j}\right] \\
& \quad+\frac{\partial}{\partial \rho_{i}} \phi\left(\rho_{i}, R_{j}\right)\left[\int _ { \mathbb { R } ^ { 2 } } w ( R _ { i } - R _ { j } ) \left(\alpha h_{1}\left(\rho_{j}-\rho_{i}\right)\right.\right. \\
& \left.\left.\quad+\beta\left\langle h_{2}\left(\rho_{j}-\rho_{i}\right)\right\rangle\right) f\left(\rho_{j}, R_{j}, \tau\right) \mathrm{d} \rho_{j} \mathrm{~d} R_{j}\right] \\
& \left.\quad+\frac{\sigma^{2}}{2} \frac{\partial^{2}}{\partial \rho_{i}^{2}} \phi\left(\rho_{i}, R_{j}\right)\left[\int_{\mathbb{R}^{2}} w\left(R_{i}-R_{j}\right) f\left(\rho_{j}, R_{j}, \tau\right) \mathrm{d} \rho_{j} \mathrm{~d} R_{j}\right]\right\} \mathrm{d} R_{i} \mathrm{~d} \rho_{i} .
\end{aligned}
$$

It remains to show that under suitable boundary conditions Eq. (47) gives the desired weak formulation of the Fokker-Planck equation. We split the boundary terms $B T$ 
into the different parts $B T_{i}, i=1,2,3$ that arises, respectively, from each integral. They are given by

$$
\begin{aligned}
B_{1} & =\int_{\mathbb{R}}\left[f\left(\rho_{i}, R_{i}, \tau\right) \phi\left(\rho_{i}, R_{i}\right)\left(\int_{\mathbb{R}^{2}} w\left(R_{i}-R_{j}\right)\left(b\left(\rho_{i}-\rho_{j}\right)-b\left(R_{i}-R_{j}\right)\right) f\left(\rho_{j}, R_{j}, \tau\right) \mathrm{d} R_{j} \mathrm{~d} \rho_{j}\right)\right]_{R_{i}=-\infty}^{R_{i}=+\infty} \mathrm{d} \rho_{i} \\
B_{2} & =\int_{\mathbb{R}}\left[f\left(\rho_{i}, R_{i}, \tau\right) \phi\left(\rho_{i}, R_{i}\right)\left(\int_{\mathbb{R}^{2}} w\left(R_{i}-R_{j}\right)\left(\alpha h_{1}\left(\rho_{j}-\rho_{i}\right)+\beta\left\langle h_{2}\left(\rho_{j}-\rho_{i}\right)\right\rangle\right) f\left(\rho_{j}, R_{j}, \tau\right) \mathrm{d} R_{j} \mathrm{~d} \rho_{j}\right)\right]_{\rho_{i}=-\infty}^{\rho_{i}=+\infty} \mathrm{d} R_{i} \\
B_{3} & =\frac{\sigma^{2}}{2} \int_{\mathbb{R}}\left[\frac{\partial}{\partial \rho_{i}} \phi\left(\rho_{i}, R_{i}\right) f\left(\rho_{i}, R_{i}, \tau\right)\left(\int_{\mathbb{R}^{2}} w\left(R_{i}-R_{j}\right) f\left(\rho_{j}, R_{j}, \tau\right) \mathrm{d} \rho_{j} \mathrm{~d} R_{j}\right)\right. \\
& \left.-\phi\left(\rho_{i}, R_{i}\right) \frac{\partial}{\partial \rho_{i}}\left[f\left(\rho_{i}, R_{i}, \tau\right)\left(\int_{\mathbb{R}^{2}} w\left(R_{i}-R_{j}\right) f\left(\rho_{j}, R_{j}, \tau\right) \mathrm{d} \rho_{j} \mathrm{~d} R_{j}\right)\right]\right]_{\rho_{i}=-\infty}^{\rho_{i}=+\infty} \mathrm{d} R_{i} .
\end{aligned}
$$

These three terms are zero, if the following boundary conditions are satisfied:

$$
\lim _{\left|R_{i}\right| \rightarrow+\infty} f\left(\rho_{i}, R_{i}, \tau\right)=0, \quad \lim _{\left|\rho_{i}\right| \rightarrow+\infty} f\left(\rho_{i}, R_{i}, \tau\right)=0, \quad \lim _{\left|\rho_{i}\right| \rightarrow+\infty} \frac{\partial}{\partial \rho_{i}} f\left(\rho_{i}, R_{i}, \tau\right)=0 .
$$

These boundary condition are guaranteed for the Boltzmann equation $f_{\gamma}\left(\rho_{i}, R_{i}, \tau\right)$ by mass conservation and the upper and lower bounds on the mean (see (14)). Therefore, (47) is the weak form of the Fokker-Planck equation

$$
\begin{aligned}
& \frac{\mathrm{d}}{d \tau} \int_{\mathbb{R}^{2}} \phi\left(\rho_{i}, R_{i}\right) f\left(\rho_{i}, R_{i}, \tau\right) \mathrm{d} R_{i} \mathrm{~d} \rho_{i}= \\
& \quad \int_{\mathbb{R}^{2}} \phi\left(\rho_{i}, R_{i}\right)\left\{-\frac{\partial}{\partial R_{i}}\left[f ( \rho _ { i } , R _ { i } , \tau ) \int _ { \mathbb { R } ^ { 2 } } w ( R _ { i } - R _ { j } ) \left(b\left(\rho_{i}-\rho_{j}\right)\right.\right.\right. \\
& \left.\left.\quad-b\left(R_{i}-R_{j}\right)\right) f\left(\rho_{j}, R_{j}\right) \mathrm{d} \rho_{j} \mathrm{~d} R_{j}\right] \\
& \quad-\frac{\partial}{\partial \rho_{i}}\left[f ( \rho _ { i } , R _ { i } , \tau ) \int _ { \mathbb { R } ^ { 2 } } w ( R _ { i } - R _ { j } ) \left(\alpha h_{1}\left(\rho_{j}-\rho_{i}\right)\right.\right. \\
& \left.\left.+\beta\left\langle h_{2}\left(\rho_{j}-\rho_{i}\right)\right\rangle\right) f\left(\rho_{j}, R_{j}\right) \mathrm{d} \rho_{j} \mathrm{~d} R_{j}\right] \\
& \left.+\frac{\sigma^{2}}{2}\left[\int_{\mathbb{R}^{2}} w\left(R_{i}-R_{j}\right) f\left(\rho_{j}, R_{j}, \tau\right) \mathrm{d} \rho_{j} \mathrm{~d} R_{j}\right] \frac{\partial^{2}}{\partial \rho_{i}^{2}}\left[f\left(\rho_{i}, R_{i}, \tau\right)\right]\right\} \mathrm{d} \rho_{i} \mathrm{~d} R_{i} .
\end{aligned}
$$

\section{References}

Albi, G., Pareschi, L., Zanella, M.: Boltzmann-type control of opinion consensus through leaders. Phil. Trans. R. Soc. A 372, 20140138 (2014)

Arnold, A., Erb, J.: Sharp entropy decay for hypocoercive and non-symmetric Fokker-Planck equations with linear drift. arXiv preprint arXiv:1409.5425 (2014)

Achleitner, F., Arnold, A., Stürzer, D.: Large-time behavior in non-symmetric Fokker-Planck equations. Riv. Mat. Univ. Parma 6, 1-68 (2015)

Bellomo, N., Herrero, M.A., Tosin, A.: On the dynamics of social conflicts: looking for the Black Swan. Kinet. Relat. Models 6(3), 459-479 (2013) 
Boudin, L., Monaco, R., Salvarani, F.: Kinetic model for multidimensional opinion formation. Phys. Rev. E 81, 036109 (2010)

Burger, M., Caffarelli, L., Markowich, P.A., Wolfram, M.-T.: On a Boltzmann-type price formation model. Proc. R. Soc. A. 469(2157), 20130126 (2013)

Burger, M., Lorz, A., Wolfram, M.-T.: On a Boltzmann mean field model for knowledge growth. SIAM J. Appl. Math. 76(5), 1799-1818 (2016)

Cercignani, C.: The Boltzmann Equation and Its Applications. Springer Series in Applied Mathematical Sciences, vol. 67. Springer, New York (1988)

Cercignani, C., Illner, R., Pulvirenti, M.: The Mathematical Theory of Dilute Gases. Springer Series in Applied Mathematical Sciences, vol. 106. Springer, New York (1994)

Cordier, S., Pareschi, L., Piatecki, C.: Mesoscopic modelling of financial markets. J. Stat. Phys. 134(1), 161-184 (2009)

Degond, P., Liu, J.-G., Ringhofer, C.: Evolution of wealth in a nonconservative economy driven by local Nash equilibria. Phil. Trans. R. Soc. A 372, 20130394 (2014)

Delitala, M., Lorenzi, T.: A mathematical model for value estimation with public information and herding. Kinet. Relat. Models 7, 29-44 (2014)

Düring, B., Toscani, G.: Hydrodynamics from kinetic models of conservative economies. Phys. A Stat. Mech. Appl. 384(2), 493-506 (2007)

Düring, B., Wolfram, M.-T.: Opinion dynamics: inhomogeneous Boltzmann-type equations modelling opinion leadership and political segregation. Proc. R. Soc. Lond. A 471, 20150345 (2015)

Düring, B., Matthes, D., Toscani, G.: Kinetic equations modelling wealth redistribution: a comparison of approaches. Phys. Rev. E 78(5), 056103 (2008)

Düring, B., Markowich, P.A., Pietschmann, J.F., Wolfram, M.-T.: Boltzmann and Fokker-Planck equations modelling opinion formation in the presence of strong leaders. Proc. R. Soc. A 465(2112), 3687-3708 (2009)

Düring, B., Jüngel, A., Trussardi, L.: A kinetic equation for economic value estimation with irrationality and herding. Kinet. Relat. Models 10(1), 239-261 (2017)

Elo, A.E.: The Rating of Chess Players, Past and Present. ISHI Press International, San Jose (1978)

Glickman, M.E., Jones, A.C.: Rating the chess rating system. Chance 12(2), 21-28 (1999)

Jabin, P.-E., Junca, S.: A continuous model for ratings. SIAM J. Appl. Math. 75(2), 420-442 (2015)

Krupp, K.: Kinetische Modelle für die Rangeinstufung von Spielern, Master thesis, WWU Münster (2016)

Motsch, S., Tadmor, E.: Heterophilious dynamics enhances consensus. SIAM Rev. 56, 577-621 (2014)

Pareschi, L., Toscani, G.: Interacting Multiagent Systems: Kinetic Equations and Monte Carlo Methods. OUP, Oxford (2013)

Pareschi, L., Toscani, G.: Wealth distribution and collective knowledge: a Boltzmann approach. Phil. Trans. R. Soc. A 372, 20130396 (2014)

Simon, J.: Compact sets in the space $L^{p}(0, T ; B)$. Ann. Mat. Pura Appl. 146, 65-96 (1986)

Torregrossa, M., Toscani, G.: Wealth distribution in presence of debts. A Fokker-Planck description. Commun. Math. Sci. 16(2), 537-560 (2018)

Toscani, G.: Kinetic models of opinion formation. Commun. Math. Sci. 4(3), 481-496 (2006)

Villani, C.: Hypocoercivity. Memoirs of the American Mathematical Society, vol. 202(950). American Mathematical Society, Providence (2009)

Zeidler, E.: Non Linear Functional Analysis and Application, vol. II/A. Springer, New York (1990) 